\title{
FOMALHAUT'S DEBRIS DISK AND PLANET: CONSTRAINING THE MASS OF FOMALHAUT B FROM DISK MORPHOLOGY
}

\author{
E. Chiang ${ }^{1,2}$, E. $\operatorname{Kite}^{2}$, P. $\operatorname{Kalas}^{1}$, J. R. Graham ${ }^{1}$, and M. Clampin ${ }^{3}$ \\ ${ }^{1}$ Department of Astronomy, 601 Campbell Hall, University of California at Berkeley, Berkeley, CA 94720, USA; echiang@astro.berkeley.edu \\ ${ }^{2}$ Department of Earth and Planetary Science, 307 McCone Hall, University of California at Berkeley, Berkeley, CA 94720, USA \\ ${ }^{3}$ Goddard Space Flight Center, Greenbelt, MD 20771, USA \\ Received 2008 September 30; accepted 2008 November 13; published 2009 March 3
}

\begin{abstract}
Following the optical imaging of exoplanet candidate Fomalhaut b (Fom b), we present a numerical model of how Fomalhaut's debris disk is gravitationally shaped by a single interior planet. The model is simple, adaptable to other debris disks, and can be extended to accommodate multiple planets. If Fom $\mathrm{b}$ is the dominant perturber of the belt, then to produce the observed disk morphology it must have a mass $M_{\mathrm{pl}}<3 M_{\mathrm{J}}$, an orbital semimajor axis $a_{\mathrm{pl}}>101.5 \mathrm{AU}$, and an orbital eccentricity $e_{\mathrm{pl}}=0.11-0.13$. These conclusions are independent of Fom b's photometry. To not disrupt the disk, a greater mass for Fom b demands a smaller orbit farther removed from the disk; thus, future astrometric measurement of Fom b's orbit, combined with our model of planet-disk interaction, can be used to determine the mass more precisely. The inner edge of the debris disk at $a \approx 133$ AU lies at the periphery of Fom b's chaotic zone, and the mean disk eccentricity of $e \approx 0.11$ is secularly forced by the planet, supporting predictions made prior to the discovery of Fom b. However, previous mass constraints based on disk morphology rely on several oversimplifications. We explain why our constraint is more reliable. It is based on a global model of the disk that is not restricted to the planet's chaotic zone boundary. Moreover, we screen disk parent bodies for dynamical stability over the system age of $\sim 100 \mathrm{Myr}$, and model them separately from their dust grain progeny; the latter's orbits are strongly affected by radiation pressure and their lifetimes are limited to $\sim 0.1$ Myr by destructive grain-grain collisions. The single planet model predicts that planet and disk orbits be apsidally aligned. Fomalhaut b's nominal space velocity does not bear this out, but the astrometric uncertainties may be large. If the apsidal misalignment proves real, our calculated upper mass limit of $3 M_{\mathrm{J}}$ still holds. If the orbits are aligned, our model predicts $M_{\mathrm{pl}}=0.5 M_{\mathrm{J}}, a_{\mathrm{pl}}=115 \mathrm{AU}$, and $e_{\mathrm{pl}}=0.12$. Parent bodies are evacuated from mean-motion resonances with Fom b; these empty resonances are akin to the Kirkwood gaps opened by Jupiter. The belt contains at least $3 M_{\oplus}$ of solids that are grinding down to dust, their velocity dispersions stirred so strongly by Fom b that collisions are destructive. Such a large mass in solids is consistent with Fom b having formed in situ.
\end{abstract}

Key words: celestial mechanics - circumstellar matter - planetary systems - planetary systems: protoplanetary disks - stars: individual (Fomalhaut)

\section{INTRODUCTION}

A common proper motion companion to Fomalhaut has been imaged by Kalas et al. (2008, hereafter K08) using the Hubble Space Telescope Advanced Camera for Surveys (HST ACS) coronagraph. Fomalhaut b (Fom b) orbits interior to the system's well-known circumstellar belt of dust (e.g., Holland et al. 1998; Kalas et al. 2005, hereafter K05, and references therein). While Fom b's ultralow luminosity leaves little doubt that it is of remarkably low mass, sitting well below the regime of brown dwarfs, the question remains: just how low is its mass?

Based on the observed broadband spectrum of Fom b, K08 estimated an upper limit on the mass $M_{\mathrm{pl}}$ of about $3 M_{\mathrm{J}}$. We recapitulate their reasoning as follows. Fom b is detected in HST's F814W $(0.7-0.9 \mu \mathrm{m})$ and F606W $(0.45-0.7 \mu \mathrm{m})$ passbands in 2006. The F606W flux is variable; the flux in 2006 was about half of that in 2004. Observations in 2005 with Keck in the $H$ band $(1.5-1.8 \mu \mathrm{m})$ and in 2008 with Gemini in L-prime $(3.2-4 \mu \mathrm{m})$ gave only upper limits.

The F814W flux (observed only in 2006; imaging was not attempted in 2004 for this passband) can be reproduced by thermal emission from a $2-4 M_{\mathrm{J}}, 200 \mathrm{Myr}$ old planet that formed by core accretion and is of supersolar metallicity (Hubickyj et al. 2005; Fortney et al. 2008). Unfortunately, this same model atmosphere underpredicts, by more than an order of magnitude, the F606W fluxes. At the same time, it overpredicts the $H$-band $3 \sigma$ upper limit by a factor of a few, and is marginally consistent with the L-prime $3 \sigma$ upper limit. From considerations outlined by $\mathrm{K} 08$, we can construct two, not entirely exclusive, hypotheses. Hypothesis one: F814W still traces planetary thermal emission, F606W is contaminated by variable $\mathrm{H} \alpha$ emission, and the atmospheric model requires revision in the $H$ band. The $\mathrm{H} \alpha$ hypothesis is inspired by variable $\mathrm{H} \alpha$ emission from chromospherically active and/or accreting low-mass stars and brown dwarfs. The puzzling brown dwarf GQ Lup B offers a possible precedent (Marois et al. 2007); in either the case of Fom b or that of GQ Lup B, the $\mathrm{H} \alpha$ luminosity necessary to explain the anomalously large F606W flux is $\sim 1 \%$ of that of the bolometric luminosity (unfortunately in neither case has an optical spectrum been taken). As for uncertainties in model exoplanet atmospheres (Fortney et al. 2008; Burrows et al. 2003), these appear greater in the $H$ band than in $\mathrm{F} 814 \mathrm{~W}$; the various models disagree with each other at near-infrared wavelengths by factors of a few. Hypothesis two: both F814W and F606W are contaminated by starlight reflected off an optically thick circumplanetary disk, and the F606W variability arises from variable disk accretion onto the planet, possibly implicating $\mathrm{H} \alpha$ again. To explain the detected fluxes in 2006 using reflected light alone, such a disk would have to be comparable in size to the orbits of Jupiter's Galilean satellites. It is not clear, however, how such a disk would survive for the system age of $\sim 100 \mathrm{Myr}$; protosatellite disks are thought to 
evolve on timescales shorter than a few Myr (e.g., Canup \& Ward 2002; Mosqueira \& Estrada 2003).

Since both hypotheses admit the possibility of additional sources of luminosity apart from planetary thermal emission, the $3 M_{\mathrm{J}}$ inferred from the observed $\mathrm{F} 814 \mathrm{~W}$ flux should be considered an upper limit. As a whole, this interpretation of Fom b's photometry is preliminary, subject to significant revision as both observations and theory improve.

An alternative route to probing the properties of Fom $b$ is to exploit the morphology of the circumstellar belt. Prior to the discovery of Fom b, this approach was taken by Quillen (2006, hereafter Q06), who built on earlier work by Wyatt et al. (1999). The striking intrinsic ellipticity of the belt of $e \approx 0.11$ can be forced by secular gravitational interaction with a single planet on a similarly eccentric orbit interior to the belt. ${ }^{4}$ Fom $b$ was predicted by Q06 to occupy an orbit of eccentricity of 0.1 and a semimajor axis of $\sim 119 \mathrm{AU}$, and to have a mass between about $2 \times 10^{-5}$ and $7 \times 10^{-5}$ of that of the central star. For a stellar mass of $M_{*}=2.3 M_{\odot}$, this range corresponds to $\sim 0.05-$ $0.2 M_{\mathrm{J}}$.

The predictions of Q06 rested on the idea that the belt inner edge, at the semimajor axis $a_{\text {inner }}=133 \mathrm{AU}$, is located at the outer boundary of Fom b's chaotic zone. The chaotic zone is a swath of space enclosing the planet's orbit in which test particle orbits are chaotic and short-lived, as a consequence of overlapping first-order mean-motion resonances (Wisdom 1980). The semimajor axes $a_{\text {chaotic }}$ that bound this chaotic zone are displaced to either side of the planet's semimajor axis $a_{\mathrm{pl}}$ by

$$
\Delta a_{\text {chaotic }}=\left|a_{\text {chaotic }}-a_{\text {pl }}\right| \approx 1.3 \mu^{2 / 7},
$$

where $\mu=M_{\mathrm{pl}} / M_{*}$. The coefficient of 1.3 arises from Wisdom's approximate scaling theory. Though (1) is derived for the case of a planet that occupies a circular orbit and that interacts with test particles on nearly circular orbits, Quillen \& Faber (2006) found that the $\mu^{2 / 7}$ scaling law also holds for a planet on a moderately eccentric orbit, interacting with particles on secularly forced eccentric orbits. Quillen \& Faber (2006) preferred, however, the coefficient of 1.5 that originates from the numerical integrations and eccentricity growth criterion of Duncan et al. (1989) ${ }^{5}$. Our work supports the assignment $a_{\text {inner }}=a_{\text {chaotic }}$ made by Q06. However, we will calculate still a third coefficient that best reproduces the HST images of Fomalhaut's belt; see our Equation (16).

The detection of Fom $b$ orbiting just interior to the dust belt appears to confirm the expectation of $\mathrm{K} 05$, made quantitative by $\mathrm{Q} 06$, that a planet is responsible for truncating the inner edge of the belt and endowing the belt with its eccentric shape. However, a closer examination reveals a potential problem with the hypothesis that Fom $b$ is solely responsible for the observed belt morphology. This picture predicts that the planet's orbit be apsidally aligned and coplanar with (perfectly nested inside) that of the belt. If that were the case, Fom b's observed position would place it somewhat past orbital quadrature, near a true anomaly of $109^{\circ}$. The problem is that

\footnotetext{
4 See the textbook by Murray \& Dermott (2000) for an introduction to secular perturbation theory, which essentially treats masses as orbit-averaged elliptical wires.

5 Duncan et al. (1989) also provided a simple explanation of behavior within the chaotic zone (for the case of circular orbits): particles residing there are perturbed so strongly by the planet at each conjunction that successive conjunctions occur at uncorrelated longitudes; consequently, the particle undergoes a random walk in semimajor axis and eccentricity, with steps in the walk corresponding to impulses at every conjunction.
}

the expected orbital velocity near quadrature (about $4.2 \mathrm{~km}$ $\mathrm{s}^{-1}$ for a stellar mass of $2.3 M_{\odot}$ and a semimajor axis of 115 $\mathrm{AU}$ ) is lower than K08's estimate for the actual, deprojected space velocity of Fom b $\left(5.5_{-0.7}^{+1.1} \mathrm{~km} \mathrm{~s}^{-1}\right)$. The large observed space velocity implies that Fom $\mathrm{b}$ is currently located closer to periastron and that planet and belt orbits are not apsidally aligned.

Should the apsidal misalignment be taken seriously and the single planet picture abandoned? It is hard to say. With Fom $b$ detected at only two epochs so far, it is impossible to derive a unique orbit. The systematic errors underlying the measured orbital velocity - in particular, frame registration errors that arise because the star is hidden behind the coronagraphic spotare difficult to quantify with confidence. Uncertainties in the stellar mass also propagate significantly into the expected orbital velocity. More precise statements about Fom b's orbit will have to await future astrometry. Our current prejudice is to say that the observational uncertainties are large enough that apsidal alignment remains a possibility. Given the observed proximity of Fom $b$ to the belt, it would be strange if Fom $b$ were not the dominant perturber (see also the last paragraph of Section 4.2).

The open issue of apsidal alignment notwithstanding, we can still place an upper limit to the mass of Fom b by assuming that it is solely responsible for shaping the belt. Broadly speaking, more planets render more of orbital phase space chaotic. Therefore, Fom b, if abetted by other planets, could have a smaller mass than that derived under the single planet assumption and still truncate the belt at its observed inner edge. The mass derived under the single planet assumption would be an upper limit.

Within the single planet picture, the published dynamical constraint on the planet's mass is $0.05 \lesssim M_{\mathrm{pl}}\left(M_{\mathrm{J}}\right) \lesssim 0.2$ (Q06). But there are several reasons to question this result, which are as follows.

1. Belt properties weakly depend on planet mass, and therefore uncertainties in the former are magnified when constraining the latter. For example, $\Delta a_{\text {chaotic }}$, which governs the relative locations of the belt inner edge and the planet, scales only as $M_{\mathrm{pl}}^{2 / 7}$. Similarly, weak powers describe how the velocity dispersion at the boundary of the chaotic zone depends on $M_{\mathrm{pl}}$; Q06 parlays this dependence into an upper limit on $M_{\mathrm{pl}}$, on the grounds that too large a velocity dispersion violates the observed sharpness of the belt's inner edge. We will see in our work that this argument does not fully capture the actual behavior because it neglects how belt particles located some distance from the chaotic zone boundary influence the observed sharpness of the edge. In other words, sharpness can only be reliably computed with a global model, and not with one that examines the chaotic zone boundary exclusively. See Figure 8 and the related discussion in Section 3.3.1.

2. The published dynamical upper mass limit is based on the purely gravitational, collisionless behavior of test particles. But the HST observations of Fomalhaut's belt are of dust grains, which are influenced by stellar radiation pressure and interparticle collisions. As explained in Section 2 (see also Strubbe \& Chiang 2006), the scattered light observations are likely dominated by the smallest grains still bound to the star. Of all the solid material orbiting Fomalhaut, such grains have the largest ratios of surface area to mass and are the most seriously affected by radiative forces and collisions. 
3. The lower dynamical mass limit is also suspect because it is premised on particles colliding indestructibly and diffusing as members of a viscous circular ring. The argument underlying the lower limit is that the planet mass cannot be too small lest dust grains diffuse into the chaotic zone by interparticle collisions, before the planet can gravitationally eject them (see also Quillen 2007). But dust particles, colliding at minimum speeds of $\sim 100 \mathrm{~m} \mathrm{~s}^{-1}$ (see our Section 2), likely shatter one another. Moreover, their trajectories are highly elliptical as a consequence of radiation pressure (Strubbe \& Chiang 2006). Their dynamics seem to be poorly described by a diffusion equation that conserves the particle number, has constant diffusivity, and assumes circular orbits.

In this work, we present a new numerical model of the Fomalhaut dust belt under the single planet assumption. It accounts not only for gravitational sculpting by Fom b, but also for the finite collisional lifetime of dust, the size distribution of grains, and radiation forces (including Poynting-Robertson (PR) drag, though it is of minor importance compared to other perturbations). Though we do not go as far as generating a model-scattered light image to compare with observations, we take the first step toward this goal by calculating the detailed shape of the belt's vertical optical depth, $\tau_{\perp}$, as a function of the semimajor axis $a$. We compare this optical depth profile with the corresponding profile of the K05 scattered light model, which in turn was directly fitted to the $H S T$ images. From this comparison, we constrain the mass and orbit of Fom b: we calculate the possible combinations of the planet mass $M_{\mathrm{pl}}$, orbital semimajor axis $a_{\mathrm{pl}}$, and orbital eccentricity $e_{\mathrm{pl}}$. These results are independent of any measurement of Fom b, and in particular of its spectrum. Only as a final extra step do we use information on Fom b's observed stellocentric distance to see if some of our mass-orbit combinations might be ruled out.

Our work corroborates the overall picture of Q06-that the planet's chaotic zone truncates the inner edge of the belt and that the planet's orbital eccentricity induces a similar mean eccentricity in the belt (Wyatt et al. 1999)—but we introduce enough improvements, especially with regards to our separate handling of unobservable parent bodies and observable dust grains, that our dynamical constraint on Fom b's mass supersedes that of Q06. Moreover, if future astrometry reveals that that there is no significant apsidal misalignment, then the precise relationship we derive between Fom b's mass and its orbit can be used to determine the former using the latter. Our model is simple, robust to uncertainties in input parameters, and applicable to other systems. Though we restrict consideration to a single interior planet, additional perturbers can be added.

In Section 2, we present an overview of the Fomalhaut belt, estimating its physical properties to order-of-magnitude accuracy. These estimates inform our choices for the input parameters of our numerical model; this model and its output are detailed in Section 3. We summarize and discuss our results-also describing the curious anomalous acceleration of Fomalhaut measured by the Hipparcos satellite, and how other planetary companions in addition to Fom b affect our conclusions-in Section 4.

\section{ORDERS OF MAGNITUDE}

We derive basic properties of the Fomalhaut dust belt, working as much as possible from first principles and direct observations. The conclusions reached in the following subsections form the basis of our numerical model in Section 3.

\subsection{Bound Dust Grains Present Absorbing, Geometric Cross Sections}

Fomalhaut is a spectral type A star of luminosity $L_{*}=$ $16 L_{\odot}$, mass $M_{*} \approx 2.3 M_{\odot}$, and age $t_{\mathrm{age}}=200 \pm 100 \mathrm{Myr}$ (Barrado y Navascues et al. 1997; Barrado y Navascues 1998). The circumstellar debris emits a fractional infrared excess of $L_{\mathrm{IR}} / L_{*}=4.6 \times 10^{-5}$ (Song et al. 2001). K05 reported that from 0.6 to $0.8 \mu \mathrm{m}$ wavelengths, the ring has a spatially integrated apparent magnitude of $m_{\text {app }}=16.2$, nearly entirely due to reflected starlight; for the star, $m_{\mathrm{app}, *}=1.12$. Therefore, the fractional reflected luminosity is $L_{\mathrm{ref}} / L_{*} \approx 10^{-6}$. If we ignore order-unity effects introduced by anisotropy in the scattering phase function (see K05 for a fit to this phase function), the dust albedo is of order $L_{\mathrm{ref}} / L_{\mathrm{IR}} \approx 0.02$-the grains are nearly purely absorbing.

Dust is generated by the collisional comminution of larger parent bodies. The largest parent bodies sit at the top of the collisional cascade and have lifetimes against collisional disruption equal to $t_{\text {age }}$. We take these largest parents to occupy a torus of radius $R \approx 140 \mathrm{AU}$, annular width $\Delta R<R$, and uniform vertical thickness $2 H<R$, to match the observations of K05. Strubbe \& Chiang (2006) referred to this torus as the "birth ring." In principle, a grain of a given size that is born into the torus is lost from it in one of four ways: collisional comminution, expulsion by radiation pressure, ejection by planetary scattering, or orbital decay by PR drag. In practice, for many debris disks, the first two channels are more important than the fourth (Wyatt 2005; Strubbe \& Chiang 2006; Thébault \& Wu 2008). The ratio of the force of radiation pressure to that of stellar gravity is

$$
\beta=\frac{3 L_{*}}{16 \pi c G M_{*} \rho s}
$$

for a geometrically absorbing grain of internal density $\rho \approx$ $1 \mathrm{~g} \mathrm{~cm}^{-3}$ and radius $s$, where $c$ is the speed of light and $G$ is the gravitational constant. Grains are unbound from the star when $\beta \gtrsim 1 / 2,{ }^{6}$ that is, when

$$
s<s_{\text {blow }} \approx \frac{3 L_{*}}{8 \pi c G M_{*} \rho}=8 \mu \mathrm{m} .
$$

Because $s_{\text {blow }}$ is much greater than the submicron wavelengths at which the star principally emits, cross-sections for absorption of radiation by bound grains are indeed practically geometric, as Equation (2) assumes.

\subsection{Radiation Pressure Delivers Grains onto Eccentric, Long-Period Orbits}

Upon release from parent bodies moving on circular orbits, bound grains move on orbits of eccentricity

$$
e=\frac{\beta}{1-\beta}=\frac{s_{\text {blow }}}{2 s-s_{\text {blow }}}
$$

and semimajor axis

$$
a=\frac{R}{1-e} .
$$

\footnotetext{
6 The critical value of $\beta=1 / 2$ strictly applies to dust grains released from parent bodies that initially move on circular orbits. For dust grains released from parent bodies moving on mildly eccentric orbits, as is the case in Fomalhaut's eccentric belt, the critical $\beta$ varies with the longitude of release,
} but is still near $1 / 2$. 
These expressions, which serve only to guide and which are not used in our more precise numerical models, ignore the parent-grain relative velocities with which grains are born, and also any eccentricity in the parent body orbit. Neither of these errors is serious for the large grain eccentricities of interest here $(e \gtrsim 1 / 3$; see Section 2.5). The main point is that radiation pressure flings smaller bound grains born in the torus onto more eccentric, longer period orbits.

\subsection{Collisions Between Grains Are Destructive}

Colliding belt particles will chip and shatter one another. For an angular orbital velocity $\Omega_{R}$ at semimajor axis $R$, the relative velocity between grains is at least as large as the vertical velocity dispersion, $\sim H \Omega_{R}=100 \mathrm{~m} \mathrm{~s}^{-1}$. To place this velocity into some perspective, we note that it is comparable to the maximum flow speeds of commercial sandblasting machines. ${ }^{7}$

As a consequence of radiation pressure, many of the grains will travel on bound orbits having eccentricities on the order of unity (Section 2.2). Accounting for orbital eccentricities (inplane velocity dispersion) only increases collision velocities, up to a maximum given by the local Kepler speed of $R \Omega_{R}=$ $4 \mathrm{~km} \mathrm{~s}^{-1}$. This maximum is comparable to elastic wave speeds in rock and will result in catastrophic shattering.

\subsection{Optical Depths: Radial and Vertical}

Since the grains present largely geometric cross sections for absorption of starlight, $L_{\mathrm{IR}} / L_{*}$ equals the fraction of the celestial sphere, centered on the star, that is subtended by dust grains:

$$
\frac{L_{\mathrm{IR}}}{L_{*}}=\frac{2 \pi R \times 2 H \times \tau_{R}}{4 \pi R^{2}}=\frac{H}{R} \tau_{R},
$$

where $\tau_{R} \ll 1$ is the radial geometric optical depth through the torus. K05 gave a model-dependent aspect ratio of $H / R=$ 0.025 ; then $\tau_{R}=1.8 \times 10^{-3}$.

The vertical optical depth (measured perpendicular to the belt midplane) is

$$
\tau_{\perp}=\tau_{R} \frac{2 H}{\Delta R}=\frac{L_{\mathrm{IR}}}{L_{*}} \frac{2 R}{\Delta R},
$$

independent of $H$. Again from the scattered light observations, $\Delta R / R \approx 0.17(\mathrm{~K} 05)$, whence $\tau_{\perp}=5.4 \times 10^{-4}$.

\subsection{Observable Grains in Fomalhaut's Belt are Bound, and \\ Their Lifetime is Set by Collisions, and Not by PR Drag}

Unbound grains, for which $\beta \gtrsim 1 / 2$, exit the torus after a local dynamical time,

$$
t_{\text {esc }}\left(s<s_{\text {blow }}\right) \sim \frac{1}{\Omega_{\mathrm{R}}} \sim 200 \mathrm{yr} .
$$

Because the lifetime of unbound grains, $t_{\mathrm{esc}}$, is much shorter than the lifetime of bound grains - the latter lifetime is set by collisional disruption; see Equation (9)-the steady-state population of bound grains will be proportionately greater than the unbound population. Combining this fact with the tendency for grain size distributions to concentrate their collective geometric cross-section on the smallest sizes, we posit that bound grains near the blow-out size, say $s_{\text {blow }}<s \lesssim 2 s_{\text {blow }}$, are responsible

\footnotetext{
7 See, for example,

http://www.nauticexpo.com/boat-manufacturer/sandblasting-machine-19911. html.
}

for much of the observed scattered light. This view is consistent with the discussion of particle sizes by K05.

Such grains occupy eccentric orbits, $e>1 / 3$, and are disrupted by collisions amongst themselves. The lifetime against collisional disruption is

$$
\begin{aligned}
& t_{\text {col }}\left(s_{\text {blow }}<s\right.\left.\lesssim 2 s_{\text {blow }}\right) \sim \frac{1}{\Omega(a) \tau} \sim \frac{1}{\Omega_{R} \tau_{R}}\left(\frac{\Delta R}{R}\right)^{1 / 2} \\
& \times \frac{1}{(1-e)^{3 / 2}} \sim 7 \times 10^{4}\left(\frac{2 / 3}{1-e}\right)^{3 / 2} \mathrm{yr},
\end{aligned}
$$

where the effective optical depth $\tau \sim \tau_{R}(R / \Delta R)^{1 / 2}$ accounts for the path length $\sim(R \Delta R)^{1 / 2}$ traversed by a grain on a highly elliptical orbit through the birth ring, where densities are highest and collisions most likely occur. Use of this path length assumes that relative grain velocities are of order the local Kepler velocity; this is an acceptable approximation for the order-unity eccentricities of interest here. An account of the limited fraction of time spent within the torus has also been made, via $\Omega(a)$ and (5).

Compare $t_{\text {col }}$ with the PR drag time,

$$
\begin{aligned}
& t_{\mathrm{PR}}\left(s_{\text {blow }}<s \lesssim 2 s_{\text {blow }}\right)=\frac{4 \pi c^{2} \rho}{3 L_{*}} E(e) R^{2} s \sim 2.5 \\
& \quad \times 10^{7} \times\left(\frac{s}{2 s_{\text {blow }}}\right)\left(\frac{2 / 3}{1-e}\right)^{1 / 2} \mathrm{yr},
\end{aligned}
$$

which is the time for an orbit of initial periastron $R$ and initial eccentricity $e$ to shrink to a point (Wyatt \& Whipple 1950). This is of the same order as the time for a grain to have its pericenter be dragged out of the birth ring, for $\Delta R$ not much less than $R$, which is the case here. The dimensionless function $E(e>1 / 3)>1.9$ quantifies the decay of orbital eccentricity and diverges as $(1-e)^{-1 / 2}$. Comparison of (9) with (10) shows that as long as $e$ is not too close to one-that is, for all particle sizes outside a tiny interval that just includes $s_{\text {blow }}$-grains are removed from the ring by collisionally cascading down to the blow-out size, and the PR drag presents only a minor loss mechanism. In other words, Fomalhaut's disk is type B or collision-dominated (Strubbe \& Chiang 2006).

Our estimate of the collisional lifetime $t_{\mathrm{col}}$ in (9) informs the duration of our dust particle simulations, introduced in Section 3.1.2.

\subsection{Total Dust Mass Versus Total Parent Body Mass}

The mass $M_{\mathrm{d}}$ in dust responsible for the scattered light is

$$
M_{\mathrm{d}} \sim \frac{8 \pi}{3} \rho s \tau_{\perp} R \Delta R \sim 10^{-3} M_{\oplus}\left(\frac{s}{2 s_{\text {blow }}}\right) .
$$

The mass $M_{\mathrm{pb}}$ in the largest parent bodies at the top of the collisional cascade is given by the steady-state condition

$$
\frac{M_{\mathrm{pb}}}{t_{\mathrm{age}}} \approx \frac{M_{\mathrm{d}}}{t_{\mathrm{col}}},
$$

which implies $M_{\mathrm{pb}} \sim 3 M_{\oplus}$. This is a minimum mass for the disk as a whole because larger bodies may still exist that are collisionless over $t_{\text {age }}$.

Some workers (e.g., Backman \& Paresce 1993) calculate the mass in parent bodies by explicitly assuming a size distribution appropriate for an idealized collisional cascade (Dohnanyi 1969) 
Table 1

Possible Properties of Fom b and Numbers of Surviving Belt Particles

\begin{tabular}{|c|c|c|c|c|c|c|c|c|c|c|c|c|}
\hline $\begin{array}{l}M_{\mathrm{pl}} \\
\left(M_{\mathrm{J}}\right)\end{array}$ & $\begin{array}{c}a_{\mathrm{pl}} \\
(\mathrm{AU})\end{array}$ & $e_{\mathrm{pl}}$ & $\begin{array}{c}N_{\mathrm{tp}} \\
(t=0)\end{array}$ & $\begin{array}{c}N_{\mathrm{tp}} \\
\left(t=10^{8} \mathrm{yr}\right)\end{array}$ & $\begin{array}{l}N_{\text {tp }} \\
(0)^{\mathrm{a}}\end{array}$ & $\begin{array}{c}N_{\text {tp }} \\
(0.00625)\end{array}$ & $\begin{array}{c}N_{\mathrm{tp}} \\
(0.0125)\end{array}$ & $\begin{array}{c}N_{\mathrm{tp}} \\
(0.025)\end{array}$ & $\begin{array}{c}N_{\text {tp }} \\
(0.05)\end{array}$ & $\begin{array}{c}N_{\text {tp }} \\
(0.1)\end{array}$ & $\begin{array}{c}N_{\mathrm{tp}} \\
(0.2)\end{array}$ & $\begin{array}{c}N_{\text {tp }} \\
(0.4)\end{array}$ \\
\hline $0.1^{b}$ & 120 & 0.115 & $10^{4}$ & 5798 & 5798 & 5796 & 5798 & 5798 & 5798 & 5798 & 5798 & 5727 \\
\hline 0.3 & 115.5 & 0.118 & $10^{4}$ & 4471 & 4471 & 4467 & 4471 & 4471 & 4471 & 4471 & 4470 & 4421 \\
\hline 1 & 109 & 0.123 & $10^{4}$ & 4191 & 4191 & 4191 & 4191 & 4191 & 4191 & 4191 & 4191 & 4187 \\
\hline 3 & 101.5 & 0.130 & $10^{4}$ & 4466 & 4466 & 4456 & 4454 & 4465 & 4465 & 4466 & 4466 & 4456 \\
\hline $10^{\mathrm{c}}$ & 94 & 0.138 & $10^{4}$ & 1548 & 1548 & 1546 & 1539 & 1433 & 1033 & 1460 & 1528 & 1548 \\
\hline
\end{tabular}

Notes.

${ }^{\text {a }}$ The pure number in parentheses for this column and subsequent columns equals $\beta$. All $\beta$-simulations tabulated here are integrated for $t_{\text {col }}=10^{5}$ yr, following the $10^{8} \mathrm{yr}$ long parent body integration.

${ }^{\mathrm{b}}$ For our $0.1 \mathrm{MJ}_{\mathrm{J}}$ run, initial parent body semimajor axes extend from $125 \mathrm{AU}$ to $140 \mathrm{AU}$

${ }^{c}$ The $10 M_{\mathrm{J}}$ run is not favored since it produces a vertical optical depth profile that does not match that of the K05 scattered light model.

and taking the upper size to be some value greater than $1 \mathrm{~km}$. Not only is it unnecessary to specify a size distribution, but it is also not justified to adopt a specific value for the parent body size without first establishing that a typical such body can be collisionally disrupted within the finite age of the system. The super-kilometer sizes often invoked (e.g., K05) fail this test.

\section{NUMERICAL MODEL}

We devise a dynamical model of the Fomalhaut planet-belt system that reproduces approximately some of the properties inferred from the HST observations. We compute the shape of the vertical optical depth profile, $\tau_{\perp}(a)$, of dust particles in the belt and match this profile against that of the K05 scattered light model. We seek in particular to find those combinations of planet mass and orbit that yield an inner edge to the belt of $a_{\text {inner }}=133 \mathrm{AU}$.

As stressed throughout this paper, the model assumes only a single interior planet apsidally aligned with the belt, an idealization not supported by Fom b's nominal space velocity (Section 1) or by the Hipparcos data (Section 4.2). Nevertheless, the planet masses derived from the single planet model can usefully be interpreted as upper limits (Section 1).

The procedure is detailed in Section 3.1, results are given in Section 3.2, and extensions of the model, including some validation tests, are described in Section 3.3.

\subsection{Procedure}

Our numerical modeling procedure is divided into four steps, described in Sections 3.1.1-3.1.4. In short, we (1) create a population of parent bodies that is stable to gravitational perturbations from Fom $\mathrm{b}$ over $t_{\mathrm{age}}$; (2) release dust particles from stable parent bodies and follow dust trajectories in the presence of radiation forces over the collisional lifetime $t_{\mathrm{col}}$; (3) compute the optical depth profile $\tau_{\perp}(a)$ of dust particles at the end of $t_{\text {col }}$, accounting for their size distribution; and (4) compare with the K05 scattered light model, which serves as our proxy for the observations.

\subsubsection{Step 1: Create Stable Parent Bodies}

Parent bodies (a) execute orbits that are stable to gravitational perturbations over $t_{\mathrm{age}}$, (b) occupy the top of the collisional cascade, which by definition implies that their orbits are little affected by catastrophic collisions for times $t<t_{\mathrm{age}}$, and (c) are large enough so that radiation forces are negligible. We further assume that (d) the self-gravity of the belt is negligible. Thus, the problem of simulating a realistic parent body is a purely gravitational, three-body problem involving the star, planet, and exterior parent body, where the parent body is treated as a test particle. Finding stable test particle orbits is a straightforward matter of specifying their initial conditions, integrating the equations of motion forward for $\sim t_{\text {age }}$, and selecting those particles that survive the integration.

Integrations of parent bodies are performed with the swift_whm code, written by $\mathrm{H}_{\text {. Levison and M. Duncan }}$ (www.boulder.swri.edu/ hal/swift.html) using the Wisdom \& Holman (1991) algorithm. We set the stellar mass $M_{*}=2.3 M_{\odot}$. In each of our five mass models, a planet of mass $M_{\mathrm{pl}} \in$ $(0.1,0.3,1,3,10) M_{\mathrm{J}}$ resides on a (fixed) elliptical orbit of the semimajor axis $a_{\mathrm{pl}}$ and eccentricity $e_{\mathrm{pl}}$. These quantities are listed in Table 1; see below for how we relate $e_{\mathrm{pl}}$ to $a_{\mathrm{pl}}$, and Step 4 (Section 3.1.4) for how we select $a_{\mathrm{pl}}$ given $M_{\mathrm{pl}}$. The planet's orbital plane defines the $x-y$ reference plane for the system. The planet's longitude of periastron $\widetilde{\omega}_{\mathrm{pl}}=0$. At the start of the integration, the planet is located at periastron. All orbital elements reported here are stellocentric and osculating unless otherwise noted.

Each run begins with $N_{\text {tp }}=10^{4}$ test particles and lasts $10^{8}$ yr. Particles are discarded as "unstable" if either they approach within a Hill sphere $R_{\mathrm{H}}=(\mu / 3)^{1 / 3} a_{\mathrm{pl}}$ of the planet or their distance from the star exceeds $1000 \mathrm{AU}$, as a result of gravitational scatterings off the planet. Particles that survive until the end of the run are deemed "stable" and are used in subsequent steps of our procedure. The integration time step is 15,000 days, equivalent to $5 \%-7 \%$ of the planet's orbital period, depending on the model.

Initial conditions for test particles are as follows. Initial semimajor axes $a$ are distributed uniformly between $120 \mathrm{AU}$ and $140 \mathrm{AU}$ (for the $0.1 M_{\mathrm{J}}$ model, the starting particle semimajor axis is $125 \mathrm{AU}$ since the planet semimajor axis turns out to be $\left.a_{\mathrm{pl}}=120 \mathrm{AU}\right)$. Initial eccentricities are set equal to the forced values given by the classical, linear, secular theory of LaplaceLagrange (L-L):

$$
e(a)=e_{\text {forced }}(a)=\frac{b_{3 / 2}^{(2)}\left(a_{\mathrm{pl}} / a\right)}{b_{3 / 2}^{(1)}\left(a_{\mathrm{pl}} / a\right)} e_{\mathrm{pl}},
$$

where $b$ 's are the usual Laplace coefficients (e.g., Murray \& Dermott 2000). Initial inclinations $i$ of test particles are drawn randomly from a uniform distribution between 0 and $0.025 \mathrm{rad}^{8}$

\footnotetext{
8 Such an inclination distribution is unphysical because in reality, there is zero probability density for finding an object with zero inclination. Nevertheless, we adopt our boxcar distribution for simplicity.
} 
Our maximum inclination matches the opening angle of the K05 scattered light disk model. Initial longitudes of periastron of all particles equal the secularly forced value $\widetilde{\omega}=0$, corresponding to apsidal alignment with the planet; longitudes of the ascending node $\widetilde{\Omega}$ are drawn randomly from a uniform distribution between 0 and $2 \pi$, and arguments of periastron $\omega=-\widetilde{\Omega}$ (so that $\widetilde{\omega}=0$ ). Finally, initial mean anomalies $M$ are drawn randomly from a uniform distribution between 0 and $2 \pi$.

For a given $a_{\mathrm{pl}}$ in a given model, the planet's eccentricity $e_{\mathrm{pl}}$ is such that a test particle at $a=140.7 \mathrm{AU}$ acquires a secularly forced eccentricity of $e=0.11$, as computed using (13). Such parameters are inspired by the elliptical orbit fitted by K05 to the brightest portions of the belt. The planetary eccentricity so chosen is about $e_{\mathrm{pl}}=0.12$, varying by up to $15 \%$ among our five models (see Table 1).

According to L-L, the initial conditions so prescribed produce test particle orbits whose eccentricity vectors $\mathbf{e}=$ $(e \cos \widetilde{\omega}, e \sin \widetilde{\omega})$ are purely forced; they have and will have no free component (e.g., Murray \& Dermott 2000). As such, because the planet's orbit is fixed, belt particle orbits also should not vary, at the L-L level of approximation. ${ }^{9}$ In Section 3.2.4, we describe the extent to which this expectation is borne out.

Our initial conditions, which comprise nested, apsidally aligned, purely forced elliptical orbits, are designed to reproduce the observed elliptical belt of Fomalhaut (Wyatt et al. 1999; Quillen 2006). However, such forced orbits are not the only ones that are stable in the vicinity of Fom b. In Section 3.3.3, we experiment with a different set of initial conditions that generate another class of stable parent body.

\subsubsection{Step 2: Integrate Dust Trajectories}

Having created an ensemble of stable parent bodies, we now model the dust generated by such bodies. At the end of a $10^{8} \mathrm{yr}$ long integration from Step 1, we take each stable parent body and have it "release" a dust grain with the same instantaneous position and velocity as its parent's. Each dust grain's trajectory is then integrated forward under the effects of radiation pressure and PR drag. That is, in addition to the gravitational accelerations from the star and the planet, a dust particle also feels a radiative acceleration (see, e.g., Burns et al. 1979)

$$
\mathbf{a}_{\mathrm{rad}}=\frac{G M_{*} \beta}{r^{2}}\left(\hat{\mathbf{r}}-\frac{v_{r} \hat{\mathbf{r}}+\mathbf{v}}{c}\right),
$$

where $\mathbf{r}=r \hat{\mathbf{r}}$ is the vector displacement from the star to the grain, $\mathbf{v}$ is the velocity of the grain relative to the star, and $v_{r}=\mathbf{v} \cdot \hat{\mathbf{r}}$ accounts for the Doppler shift in stellar radiation seen by the grain. We add this radiative acceleration to the Bulirsch-Stoer (B-S) integrator swift_bs, written by Levison \& Duncan. We prefer to modify the B-S integrator over the Wisdom-Holman integrator, since the latter is designed to model a dissipationless Hamiltonian system; when PR drag is included, the system is dissipative, and it is not obvious to us how we

\footnotetext{
9 L-L truncates the secular disturbing function at $O\left(e^{2}, i^{2}\right)$ and so in reality and in numerical integrations, the eccentricities and apsidal angles are still expected to vary somewhat with time with our initial conditions, even if the system were purely secular. One can avoid such a truncation error by employing Gauss's perturbation equations for $\dot{e}$ and $\dot{\widetilde{\omega}}$ and integrating the planetary forces over Gaussian wires (e.g., Murray \& Dermott 2000), thereby finding exact forced eccentricities for which $\dot{e}=\dot{\widetilde{\omega}}=0$. We skip this refinement, in part because the system is not purely secular; nearby mean-motion resonances influence the dynamics.
}

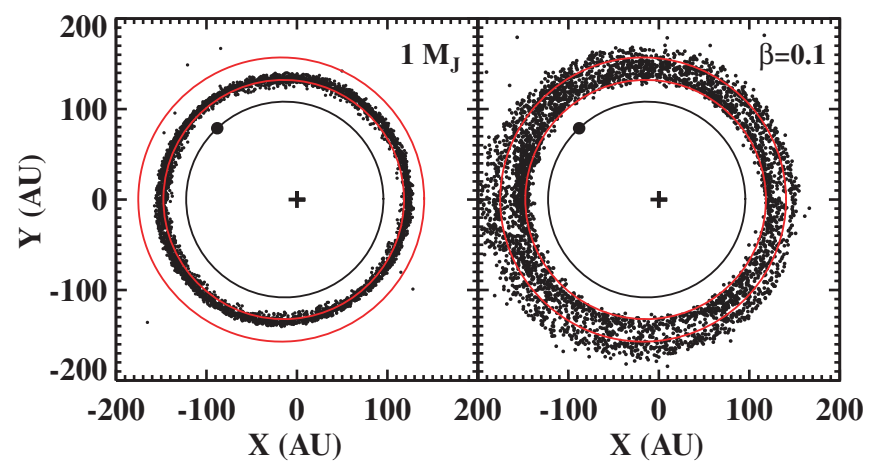

Figure 1. Snapshots of parent bodies (left) and $\beta=0.1$ dust grains (right), for our $1 M_{\mathrm{J}}$ model. The cross marks Fomalhaut, while the solid circle marks Fomalhaut $\mathrm{b}$. Parent bodies are imaged after an integration time of $t_{\mathrm{age}}+t_{\mathrm{col}}$. Dust particles are released from parent bodies with zero relative velocity after $t_{\text {age }}$ and their trajectories integrated forward with $\beta=0.1$ for $t_{\mathrm{col}}$. Red ellipses correspond to the inner and outer boundaries of the K05 scattered light model $\left(a_{\text {inner }}=133 \mathrm{AU}, a_{\text {outer }}=158 \mathrm{AU}, e=0.11\right)$. Radiation pressure spreads dust particles outward from where they were born, but leaves their inner boundary practically coincident with that of parent bodies.

should add the radiative perturbations to the symplectic kickdrift-kick algorithm. (Nevertheless, adding radiative forces to symplectic integrators is standard practice in the literature and has been shown to produce accurate results.) Though the B-S integrator is slower, it is fast enough for our purposes since our integration times for Step 2 are short (see below). The accuracy parameter "eps" of swift_bs is set to $10^{-8}$. The modified code was tested on test particles having various $\beta$ 's, producing results for radiation blow-out and PR drag in excellent agreement with analytic and semianalytic studies (e.g., Wyatt \& Whipple 1950).

From each of the five parent body integrations completed in Step 1, we generate eight dust simulations in Step 2, each characterized by a single value of $\beta \in(0,0.00625,0.0125,0.025$, $\ldots, 0.4)$. Dust grains released with such $\beta$ 's are mostly still bound to the star, albeit on highly eccentric orbits for $\beta$ approaching 0.4 . Bound grains contribute substantially, if not predominantly, to the scattered light observations: as sketched in Section 2, because $t_{\mathrm{PR}} \gg t_{\mathrm{col}} \gg t_{\mathrm{esc}}$, the lifetime of bound grains is set by destructive interparticle collisions, and not by PR drag, and the steady-state population of bound grains greatly outweighs that of unbound grains, by $t_{\mathrm{col}} / t_{\mathrm{esc}}$.

Because the dust lifetime is set by collisions, we extract dust grain orbits for further analysis in Step 3 after integrating for a time $t_{\text {col }}$. Following our order-of-magnitude estimate (9), we set $t_{\mathrm{col}}=10^{5} \mathrm{yr}$. During the integration, we discard particles that approach within a Hill sphere of the planet or whose distances from the star exceed 10,000 AU (this is a factor of 10 larger than the cut imposed in Step 1, because large apastron distances result from the onset of radiation pressure and do not necessarily imply orbital instability). As Table 1 indicates, few if any dust particles are discarded in our $\beta$-simulations, with the exception of our $10 M_{\mathrm{J}}$ model.

In Section 3.3.2, we test the sensitivity of our results to $t_{\text {col }}$, whose value we know only within factors of a few. In that subsection, we also examine whether our results change significantly if we model the dust more realistically by releasing grains gradually over $t_{\mathrm{col}}$.

Figure 1 provides sample snapshots of dust grains and their parent bodies projected onto the $x-y$ plane, for our $1 M_{\mathrm{J}}$ model. 


\subsubsection{Step 3: Compute Optical Depth Profile}

If we had a sufficiently large number of dust particles, we could simply take a snapshot of each Step $2 \beta$-simulation after $t_{\mathrm{col}}$ and count the number of dust particles per unit $x-y$ area of the belt. We would thereby measure the surface density $N_{\beta}(x, y)$ and, by extension, the vertical optical depth $\tau_{\perp}(x, y)$. In practice, we do not have enough particles, and such an exercise produces noisy results.

We greatly improve the signal to noise by spreading each dust particle along its orbit according to how much time it spends traversing a given segment of its orbit. In other words, we replace each dust particle with its equivalent Gaussian wire, and measure the optical depth presented by the collection of wires. We refer the Kepler elements of a dust particle's orbit to $(1-\beta)$ times the stellar mass; otherwise the elements would not remain constant in a two-body approximation.

First, we construct an eccentric grid by partitioning the $x-y$ plane into a series of nested, confocal ellipses, all having the same eccentricity of 0.11 (K05), and having semimajor axes running uniformly from $a_{1}=100 \mathrm{AU}$ to $a_{N}=220 \mathrm{AU}$ in steps of $\Delta a=0.5 \mathrm{AU}$. Our goal is to measure $\tau_{\perp}\left(a_{i}\right)$, the average optical depth between the $i$ th ellipse having a semimajor axis $a_{i}$ and the $(i+1)$ th ellipse having a semimajor axis $a_{i+1}=a_{i}+\Delta a$. Each dust particle orbit is divided into 1000 segments spaced uniformly in true anomaly from 0 to $2 \pi$. Each segment maps to a certain location on the grid (i.e., the $x-y$ position of each segment falls between two adjacent ellipses on the grid). Associated with each segment is an orbital weight, equal to the fraction of the orbital period spent traversing that segment (the sum of all orbital weights for a given particle/orbit equals 1). The orbital weight for each segment is added to its grid location. This process is repeated over all segments of all orbits. Finally, at each grid location $a_{i}$, the sum of orbital weights is divided by the area of the annulus extending from the $i$ th ellipse to the $(i+1)$ th ellipse. This yields the relative surface density profile $N_{\beta}\left(a_{i}\right)$, for a simulation characterized by $\beta$.

The various $N_{\beta}$ profiles for our $1 M_{\mathrm{J}}$ model are plotted in the top panel of Figure 2. The profiles are normalized to the peak of the $N_{0}(\beta=0)$ profile. Since the number of dust particles is practically constant across all $\beta$-simulations within a given mass model (Table 1), the decreasing height of each $N_{\beta}$ profile with increasing $\beta$ simply reflects how dust particle orbits become increasingly eccentric and elongated with increasing $\beta$ (see Equation (4)). In other words, the same number of particles is being spread into a disk that extends farther out the greater the radiation pressure. At the same time, the peaks of the $N_{\beta}$ profiles hardly move with increasing $\beta$ : as long as the grain is still bound to the star, it must always return to the same stellocentric distance at which it was released, no matter how strongly it feels radiation pressure. This release distance is located in the birth ring (Strubbe \& Chiang 2006) or, more accurately, the birth ellipse of parent bodies, near $a \approx 130-140$ AU; see the left-hand panel of Figure 1.

Once all the $N_{\beta}$ profiles are in hand, we combine them linearly to produce the total optical depth profile $\tau_{\perp}$ :

$$
\begin{aligned}
\tau_{\perp}= & \sum_{\beta \neq 0} N_{\beta} \frac{\max N_{0.00625}}{\max N_{\beta}}\left(\frac{\beta}{0.00625}\right)^{q-3} \\
& +N_{0} \frac{\max N_{0.00625}}{\max N_{0}}(1+\sqrt{2}) .
\end{aligned}
$$

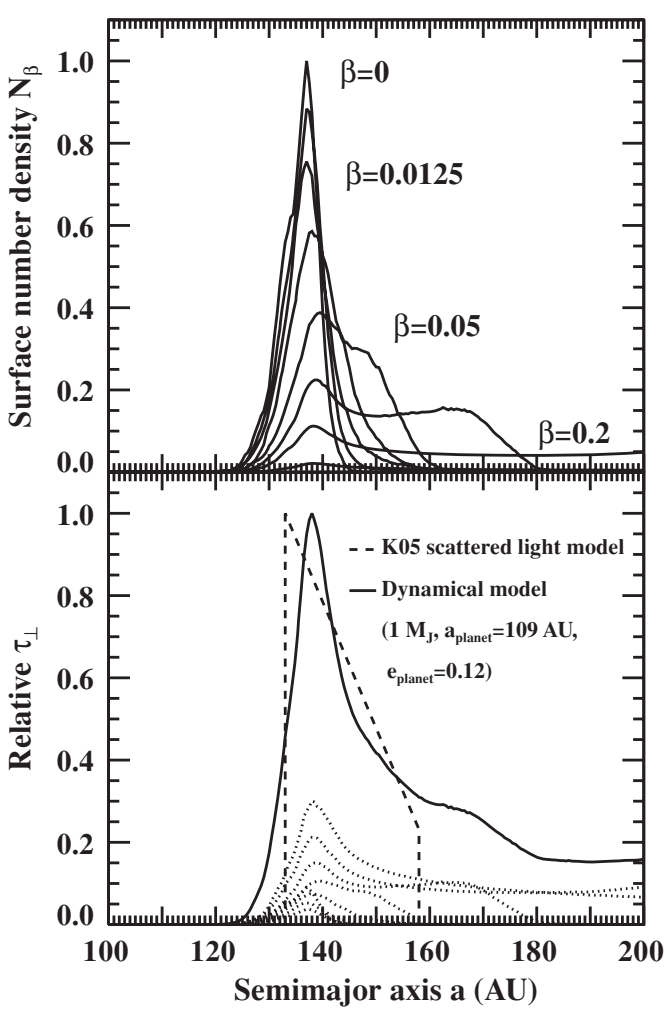

Figure 2. Top panel: surface density profiles of dust grains for our $1 M_{\mathrm{J}}$ model, computed $t_{\mathrm{col}}=10^{5} \mathrm{yr}$ after release, normalized to the peak of the $\beta=0$ curve. These profiles are computed by binning wire segments on a fixed elliptical grid, as described in the main text under Step 3 of our procedure. Profiles shrink vertically and widen horizontally with increasing $\beta$, reflecting how increased radiation pressure spreads particles outward by amplifying apastron distances. By contrast, periastron distances are more nearly conserved for bound particles, since they always return to the birth ring regardless of the strength of radiation pressure. Thus, the peaks of the $N_{\beta}$ profiles, which mark the location of the birth ring of parent bodies (see the left panel of Figure 1), hardly shift with $\beta$. Bottom panel: vertical optical depth profile (solid line) obtained by adding together the individual $N_{\beta}$ profiles (dotted lines), appropriately weighted according to a Dohnanyi size distribution (see Equation (15)). At $a \lesssim 160 \mathrm{AU}$, the profile resembles that of the K05 scattered light model (dashed line), which itself is an approximate, idealized, and nonunique representation of the HST observations. Discrepancies at large $a \gtrsim 160 \mathrm{AU}$ are expected, in large part because the $H S T$ images are too noisy to usefully constrain the K05 model there.

The rationale behind this formula is as follows. As Figure 2 indicates, the maxima of the $N_{\beta}$ profiles are all situated in the birth ring. Following Strubbe \& Chiang (2006), we posit that the size distribution of grains in the birth ring is given by a Dohnanyi (1969) cascade, with a differential power-law index $q=7 / 2$. For such a power-law size distribution, the collective surface area or geometric optical depth, evaluated per logarithmic bin in $\beta$, scales as $\beta^{q-3}\left(\propto s^{3-q}\right)$. The two factors multiplying $N_{\beta}$ in the sum in (15) enforce this scaling in the birth ring (whose location is traced by the maxima of the surface density profiles), and we have adopted the $\beta=0.00625$ bin as our reference bin.

The last term proportional to $N_{0}$ accounts for the optical depth contributed by grains having $0<\beta<0.00625$. We take the surface density profile of such grains to be given by $N_{0}$; this is a good approximation, as there is little difference between $N_{0.00625}$ and $N_{0}$ (Figure 2, top panel). Since our grid of models for $\beta \geqslant 0.00625$ is logarithmic in $\beta$-successive bins are separated by factors of $B=2$-we extend the same logarithmic grid for $\beta<0.00625$. Then the optical depth coming from all grains having $\beta<0.00625$, scaled to the optical depth in our $\beta=0.00625$ reference bin, is $\sum_{j=1}^{\infty}(1 / B)^{j(q-3)}=1+\sqrt{2}$. 


\subsubsection{Step 4: Compare with Observations}

A rigorous comparison with observations would require us to produce a scattered light image based on our dynamical model. This is a considerable task. Our $\tau_{\perp}$ profile, combined with the vertical density distribution and a grain scattering phase function, would be used to calculate the direction-dependent emissivity of the belt as a function of the three-dimensional position. This emissivity model would then be ray-traced at a nonzero viewing angle to produce a model-scattered light image. Various parameters (e.g., normalization of $\tau_{\perp}$, grain scattering asymmetry parameter, viewing angle) would need to be adjusted, including input parameters from Steps 1-3 (distribution of initial semimajor axes, distribution of initial inclinations), to produce a good subtraction of the observed image.

In this first study, we attempt none of this. Instead, we compare the $\tau_{\perp}$ profile given by (15) with the corresponding optical depth profile of the K05 scattered light model, focusing on the one belt property that seems most diagnostic of the planet mass and orbit: the belt's inner edge. The K05 optical depth profile extends from $a_{\text {inner }}=133 \mathrm{AU}$ to $a_{\text {outer }}=158 \mathrm{AU}$ and falls as $a^{-8.5}$ (their fitted volumetric number density of grains falls as $a^{-9}$, while the fitted vertical thickness of the belt increases as $a^{0.5}$ ). In our dynamical modeling, for given planet mass $M_{\mathrm{pl}}$, we adjust only the planet semimajor axis $a_{\mathrm{pl}}$ and repeat Steps 1-3 until the minimum semimajor axis at which $\tau_{\perp}$ reaches half its maximum value - the "half-maximum radius"equals $a_{\text {inner }}=133 \mathrm{AU}$.

Since our dynamical models are anchored to $a_{\text {inner }}$, we should have a sense of the uncertainty in this parameter. The K05 model is based on fits "by eye." From the visual fits, the uncertainty is about $\pm 1 \mathrm{AU}$, slightly larger than the size of a 2 pixel resolution element $(0.1$ arcsec or $0.77 \mathrm{AU}$; the images are binned $2 \times 2$ before they are modeled, to increase signal to noise). The error in $a_{\text {inner }}$ propagates into our constraints on planet mass.

It is well to appreciate that while our general goal is to reproduce the shape of the optical depth profile of the K05 scattered light model, that model is itself highly idealized, characterized by knife-edge sharp inner and outer edges and simple power-law behavior. Fitting by eye means the model is at best approximate. And as K05 cautioned, only a restricted azimuth of the belt (near their quadrant "Q2") was analyzed in detail to produce their fit parameters. Therefore, we should neither aim for, nor expect, perfect agreement between our dynamical model and the K05 model. Our task instead is to use the K05 model as a guide, to identify robust trends, and to rule out those regimes of parameter space for Fom $b$ that give blatantly poor agreement with observed belt properties.

Lastly, neither our dynamical model nor the K05 scattered light model should be trusted at large $a \gtrsim 160 \mathrm{AU}$. At large distance, barely bound grains whose $\beta$ 's are arbitrarily close to the blow-out limit of $\sim 0.5$ dominate $\tau_{\perp}$. Our set of eight $\beta$-simulations lacks the resolution in $\beta$ to accurately model this outer disk (see Strubbe \& Chiang 2006 for an analytic treatment appropriate for a circular birth ring). Observationally, the HST images at stellocentric distances $\gtrsim 158 \mathrm{AU}$ are dominated by the sky background; see Figure 3 of K05.

\subsection{Results}

In Section 3.2.1, we give an approximate formula relating the planet mass to the width of the chaotic zone, based on our five mass models. In Section 3.2.2, we compare our optical depth

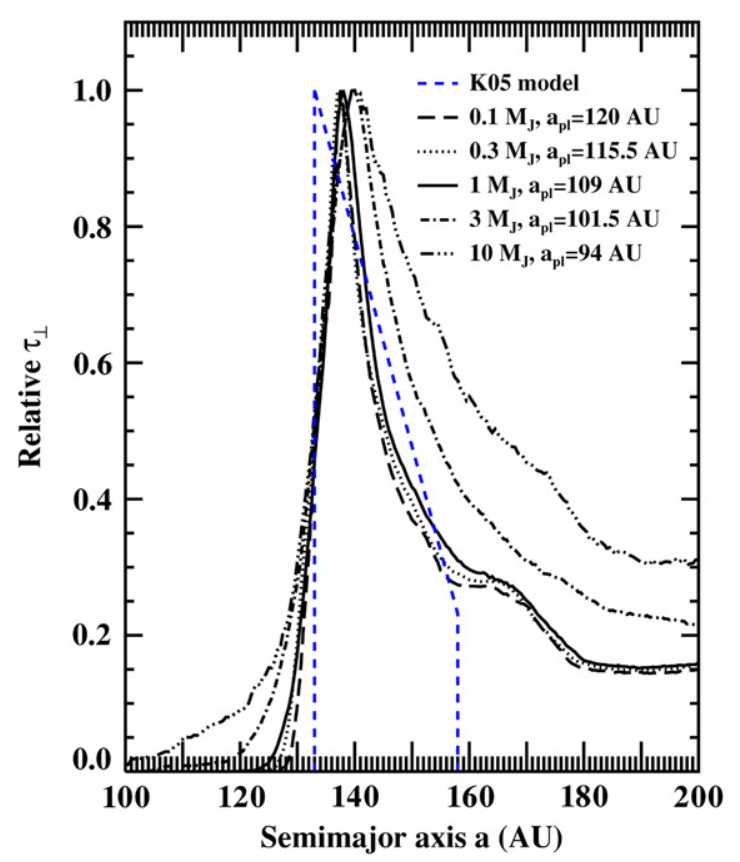

Figure 3. Vertical optical depth profiles of our five mass models (black lines), overlaid with that of the K05 scattered light model (blue dashed line). Parameters for our dynamical models are listed in the inset and are such that the "halfmaximum radius" - the minimum semimajor axis for which $\tau_{\perp}$ attains half its maximum value-equals $133 \mathrm{AU}$, the innermost semimajor axis of the K05 model. Models for which $M_{\mathrm{pl}} \leqslant 1 M_{\mathrm{J}}$ do equally well in reproducing the $\mathrm{K} 05$ model. As $M_{\mathrm{pl}}$ increases, the $\tau_{\perp}$ profiles widen because the planet increasingly perturbs dust grains onto eccentric orbits. The $10 M_{\mathrm{J}}$ model is probably unacceptably wide. At $a \gtrsim 160 \mathrm{AU}$, neither the dynamical model nor the K05 model is trustworthy; the former suffers from poor resolution in $\beta$, while the latter is limited by sky background (see Figure 3 of K05).

profiles with that of the K05 scattered light model. Based on this comparison, we argue against large planetary masses for Fom b. In Section 3.2.3, we argue the same point by comparing our model planetary orbits with the observed stellocentric distance of Fom b. Finally, the underlying dynamics of parent bodies and of dust particles is briefly discussed in Section 3.2.4.

\subsubsection{Chaotic Zone Width}

In Figure 3, we overlay the $\tau_{\perp}$ profiles of our five mass models together with the optical depth profile of the K05 scattered light model. As described in Section 3.1.4, the planet semimajor axis $a_{\mathrm{pl}}$ for each mass model is chosen such that the "half-maximum radius" - the smallest semimajor axis at which $\tau_{\perp}$ attains half its peak value-equals K05's $a_{\text {inner }}=133$ AU. $a_{\mathrm{pl}}$ 's so determined are listed in Table 1 and also annotated in Figure 3. They are such that the semimajor axis separation between the belt inner edge and planet is given by

$$
a_{\mathrm{inner}}-a_{\mathrm{pl}}=2.0 \mu^{2 / 7} a_{\mathrm{pl}},
$$

with less than $6 \%$ variation in the coefficient across mass models, and where $a_{\text {inner }}=133 \mathrm{AU}$. Because we connect more directly to the $H S T$ observations, our Fomalhaut-specific coefficient of 2.0 is preferred over the smaller coefficients cited by previous works; accuracy matters for determining the planet mass, whose value scales strongly as the $7 / 2$ power of distance measurements. Measured in Hill radii (evaluated using $a_{\mathrm{pl}}$ ), the separation $a_{\text {inner }}-a_{\mathrm{pl}}$ ranges from 3.7 to $4.5 R_{\mathrm{H}}$ in order of decreasing $M_{\mathrm{pl}}$. 


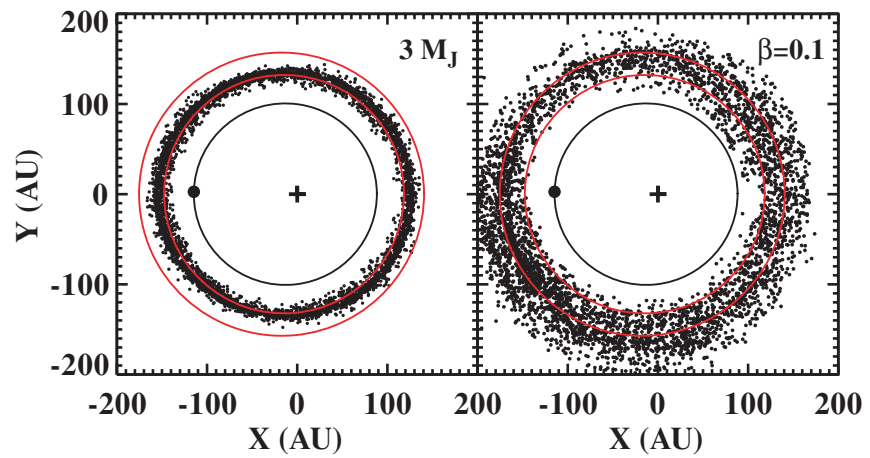

Figure 4. Snapshots of parent bodies (left) and $\beta=0.1$ dust grains (right), for our $3 M_{\mathrm{J}}$ model. Compare with the $1 M_{\mathrm{J}}$ model shown in Figure 1 . The larger the planet mass, the more the dust particles are perturbed by the planet into a more spatially extended disk.

\subsubsection{Comparison of $\tau_{\perp}$ Profiles Implies $M_{\mathrm{pl}}<3 M_{\mathrm{J}}$}

What resemblance there is in Figure 3 between dynamical and scattered light $\tau_{\perp}$ profiles leads us to believe that we are on the right track toward understanding the underlying properties of the Fomalhaut planet-belt system. We are especially encouraged when we consider that with the exception of $a_{\mathrm{pl}}$, none of our model parameters (e.g., grain size index $q$, distribution of initial semimajor axes) has been adjusted from its naive standard value. And as we emphasized at the end of Section 3.1.4, the K05 scattered light model is itself highly idealized and approximate, and does not uniquely represent the observations. In particular, the K05 model idealizes the inner edge as a step function, but smoother profiles also seem possible; the degree of smoothness may help to constrain $q$.

Discrepancies at large $a \gtrsim 160 \mathrm{AU}$ are not serious, since both the dynamical and scattered light models are known to fail there (Section 3.1.4). The deficiency in our dynamical model can be remedied by having a finer grid in $\beta$ near the blow-out value of $\sim 0.5$, while improvements in the scattered light model await deeper imaging campaigns.

As $M_{\mathrm{pl}}$ increases, the $\tau_{\perp}$ profiles broaden-see, in particular, the curve for $10 M_{\mathrm{J}}$. Upon release, dust particles find themselves in a weakened stellar potential because of radiation pressure. More massive planets more readily perturb dust onto more eccentric orbits that extend both inside and outside of the birth ring. This is further illustrated in Figure 4, which shows a snapshot of $\beta=0.1$ grains for $M_{\mathrm{pl}}=3 M_{\mathrm{J}}$. Moreover, as documented in Table 1, planetary perturbations are so severe for our $10 M_{\mathrm{J}}$ model that about $1 / 3$ of the dust particles launched with $\beta=0.05$ are discarded as unstable within $t_{\text {col }}=10^{5}$ yr. (Why $\beta=0.05$ ? Larger $\beta$ grains are, upon release, repelled immediately away from the planet onto highly eccentric trajectories by radiation pressure alone and are, therefore, less likely to be rendered unstable by the planet. Smaller $\beta$ grains share essentially the same stability properties as the parent bodies.)

The $\tau_{\perp}$ profile for $M_{\mathrm{pl}}=10 M_{\mathrm{J}}$ is probably unacceptably broad: at $140 \mathrm{AU} \lesssim a \lesssim 160 \mathrm{AU}$, the dynamical model predicts too large an optical depth compared with the K05 model, by about a factor of 2 . At these distances, a factor of 2 overluminosity in the belt is not easily accommodated, as judged from the error bars on the observed surface brightness profilesee Figure 3 of K05. This same $10 M_{\mathrm{J}}$ model also produces a tail extending inward to $a \lesssim 120 \mathrm{AU}$, but the observations, whose dynamic range in surface brightness is limited to less than 10:1 (see Figure 3 of K05), probably cannot rule out such a feature. We have verified that the excessively large $\tau_{\perp}$ at $a \gtrsim 140 \mathrm{AU}$ primarily follows from the large eccentricities acquired by $\beta \approx 0.2-0.4$ dust particles from planetary perturbations. By contrast, the $M_{\mathrm{pl}} \leqslant 1 M_{\mathrm{J}}$ models, which produce practically identical $\tau_{\perp}$ profiles, appear compatible with the K05 model, given the various limitations of the latter.

The low-mass models having $M_{\mathrm{pl}} \leqslant 1 M_{\mathrm{J}}$ have inner edges that are practically identically sharp. A measure of the sharpness is the distance over which $\tau_{\perp}$ rises from the half-maximum radius to the semimajor axis at which $\tau_{\perp}$ peaks, normalized by the half-maximum radius. This fractional distance is $\delta=$ 4.5 AU/133 AU $=0.034$. Quillen (2006) recognized that in fitting the observed drop-off in surface brightness for a belt that is inclined to our line of sight (LOS), a trade-off exists between the belt's vertical and radial density profiles. The belt can either have a finite vertical thickness and be knife-edge sharp in the radial direction (as in the K05 scattered light model), or have zero vertical thickness and drop off gradually in the radial direction, or be characterized by some intermediate combination. In this context, our measure for the fractional radial drop-off distance, $\delta=0.034$, compares promisingly with the fractional vertical drop-off distance inferred by $\mathrm{K} 05$, $2 H / R=0.025$. In the future, we will have to adjust both radial and vertical drop-off distances to better reproduce the scattered light observations. The vertical thickness of the belt appears to be fairly easily adjusted in our model, given that the inclinations of our dust particles are mostly unchanged from the assumed initial inclinations of our parent bodies (see Figure 7 and related discussion in Section 3.2.4).

In the bottom panel of Figure 2, we plot the separate terms in Equation (15) that add up to $\tau_{\perp}$ as dotted curves. The terms corresponding to larger $\beta$ (or smaller grain size $s \propto 1 / \beta$ ) dominate, as a consequence of our assumption that the grain size distribution in the birth ring follows a Dohnanyi law. This law apportions the bulk of the geometric surface area in the smallest grains.

\subsubsection{Fom b's Current Stellocentric Distance Implies $M_{\mathrm{pl}}<3 M_{\mathrm{J}}$}

Each of our mass models specifies a certain orbit for Fom b (Table 1) that is tuned, through multiple iterations of Steps $1-4$, to generate the observed ellipticity of the belt and to yield a half-maximum radius equal to $\mathrm{K} 05$ 's $a_{\text {inner }}=133 \mathrm{AU}$. If the apocentric distance of a model orbit is less than the observed stellocentric distance of Fom b, then that model can be ruled out.

Currently, only the distance between Fom b and its host star projected onto the sky plane is known. In 2006, that projected distance was 97.6 AU. Inferring the true stellocentric distance requires that we deproject the orbit of Fom b. But with only two epochs of observation, a unique deprojection is not possible. Nevertheless, we can perform a rough deprojection by making a few assumptions. We assume that the planet's orbit is oriented such that the line of nodes between the planet's orbit and the sky plane coincides with the line joining the observed belt ansae. We also assume that the inclination of the planet's orbit to the sky plane equals 65.6 , the same as that inferred for the belt orbital plane (K05). These assumptions do not restrict the apsidal orientation of the planet's orbit.

The resultant deprojected stellocentric distance of Fom b in 2006 is $119 \mathrm{AU}$, with a systematic error of probably no more than a couple of AUs. Such a distance argues against our $10 M_{\mathrm{J}}$ 


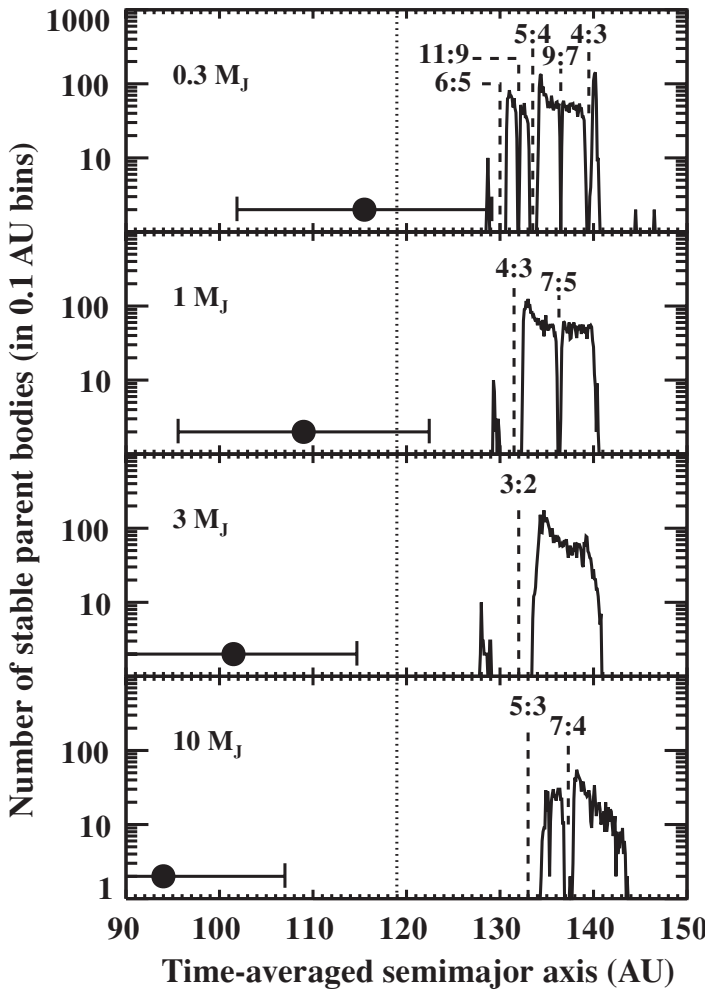

Figure 5. Histogram of time-averaged semimajor axes of parent bodies that survive for $10^{8} \mathrm{yr}$ in the vicinity of Fom $\mathrm{b}$. The bin width is $0.1 \mathrm{AU}$, and the time average is performed over $10^{5} \mathrm{yr}$. Each panel corresponds to a different mass model, as annotated. The black circle in each panel marks the semimajor axis of Fom b, with error bars extending from the model orbit's pericentric distance to its apocentric distance. Only model orbits corresponding to $M_{\mathrm{pl}} \lesssim 1 M_{\mathrm{J}}$ are consistent with the estimated deprojected stellocentric distance of Fom b in 2006 (dotted vertical line). Stable parent bodies are located outside the planet's chaotic zone, at semimajor axes greater than the planet's by about $2 \mu^{2 / 7} a_{\mathrm{pl}}$. Inside the chaotic zone, first-order mean-motion resonances overlap and particle orbits are short-lived. Outside the chaotic zone, parent bodies reside stably on secularly forced eccentric orbits, with occasional gaps located at mean-motion resonances as indicated. The gaps are evacuated for reasons likely analogous to why the solar system's Kirkwood gaps are empty of asteroids.

model, for which the apocentric distance of Fom b is $107 \mathrm{AU}$. The discrepancy is depicted in Figure 5. This same figure shows that the $3 M_{\mathrm{J}}$ model is also inconsistent, but only marginally so, and without a proper deprojection, we hesitate to rule it out. Lower masses $M_{\mathrm{pl}} \leqslant 1 M_{\mathrm{J}}$ are, by contrast, easily compatible. These findings reinforce those based purely on a comparison of the optical depth profiles (Section 3.2.2).

\subsubsection{Parent Body and Dust Particle Dynamics}

In Figure 5, we supply sample histograms of time-averaged semimajor axes of stable parent bodies generated in Step 1. The time average is performed over a $10^{5} \mathrm{yr}$ long window (with $\beta=0$ ) following each $10^{8} \mathrm{yr}$ long integration. Intriguingly, parent bodies appear evacuated from exterior mean-motion resonances established by Fom b, even outside the planet's main chaotic zone. The reasons for this are likely analogous to why the solar system's Kirkwood gaps, located at Jupiter's interior mean-motion resonances, are empty of asteroids (Wisdom 1982, 1983, 1985). A study of this phenomenon, which depends on the nonzero eccentricity of the planet's orbit, is deferred to future work.

Figure 6 plots the eccentricity vectors of the parent bodies at the end of $10^{8} \mathrm{yr}$ (black points). While they remain clustered near

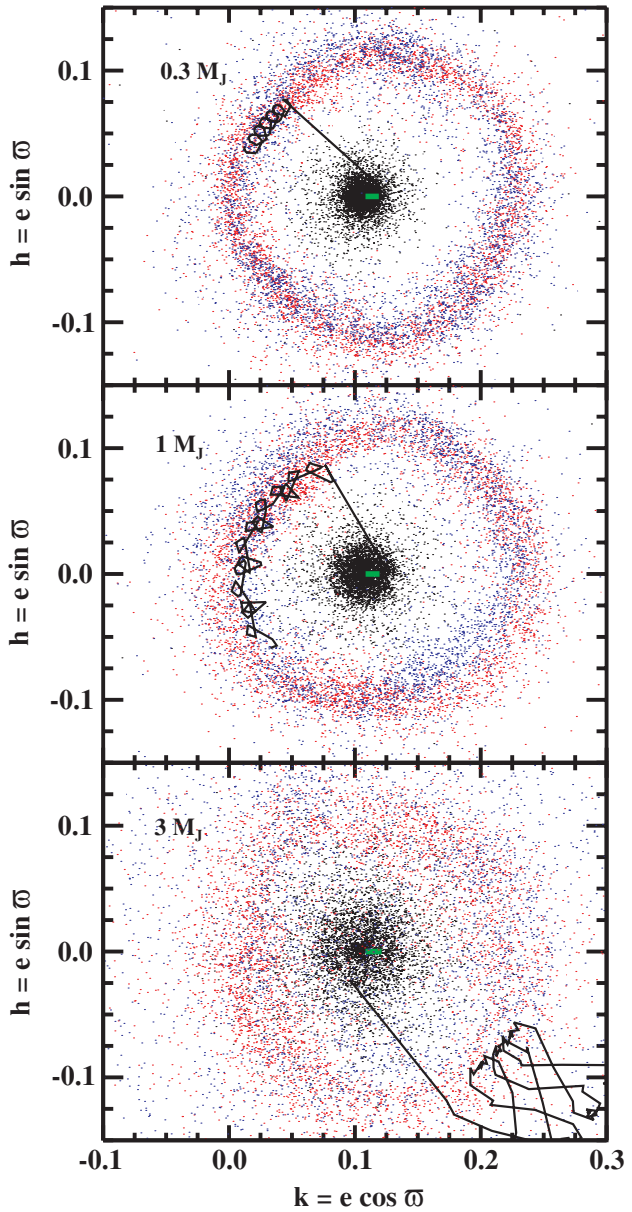

Figure 6. Locations in complex eccentricity space of all parent bodies at $t=0$ (green bar) and those parent bodies that survive for $t=10^{8} \mathrm{yr}$ (black points). The locus of survivors is not exactly centered on the locus of initial conditions because the survivors are all at large semimajor axes (smaller forced eccentricities), and because of inaccuracies in the L-L theory that was used to determine the initial conditions. Surviving parent bodies remain approximately apsidally aligned with the planet's orbit, deviating by less than $\pm 15^{\circ}$ in most cases. The deviations, that is, the dispersions in free eccentricity, increase with $M_{\mathrm{pl}}$. This same behavior was found by Quillen (2006). However, in contrast to that work, we find that the increased dispersion does not necessarily imply a more spatially diffuse inner edge to the belt; see Figure 8 and Section 3.3.1. Also shown are $\beta=0.1$ dust particles $10^{4} \mathrm{yr}$ after release (red points) and those same dust particles $10^{5} \mathrm{yr}$ after release (blue points). The trajectory of a typical dust particle is shown sampled every $10^{3} \mathrm{yr}$, starting from release. Note the large eccentricity variations for the $3 M_{\mathrm{J}}$ model.

their initially purely forced values, there is a dispersion that is not predicted by L-L: the parent bodies acquire free eccentricities despite having none to start with. The more massive the planet, the greater the free eccentricities that develop. This same behavior was found by Quillen (2006) and Quillen \& Faber (2006), who attributed it to forcing by a mean-motion resonance just outside the chaotic zone.

Figure 6 also describes how dust particles (colored points) born from parent bodies acquire free eccentricities. Initial free eccentricity vectors are distributed roughly axisymmetrically about the forced eccentricities. The initial free phase depends on the orbital phase of release. For example, a dust particle released at the parent body's periastron gains a free eccentricity vector in the $+\hat{\mathbf{k}}$ direction (total eccentricity increases), while release at apastron yields a free eccentricity in the $-\hat{\mathbf{k}}$ direction (total eccentricity decreases, at least for $\beta$ not too large). Though 


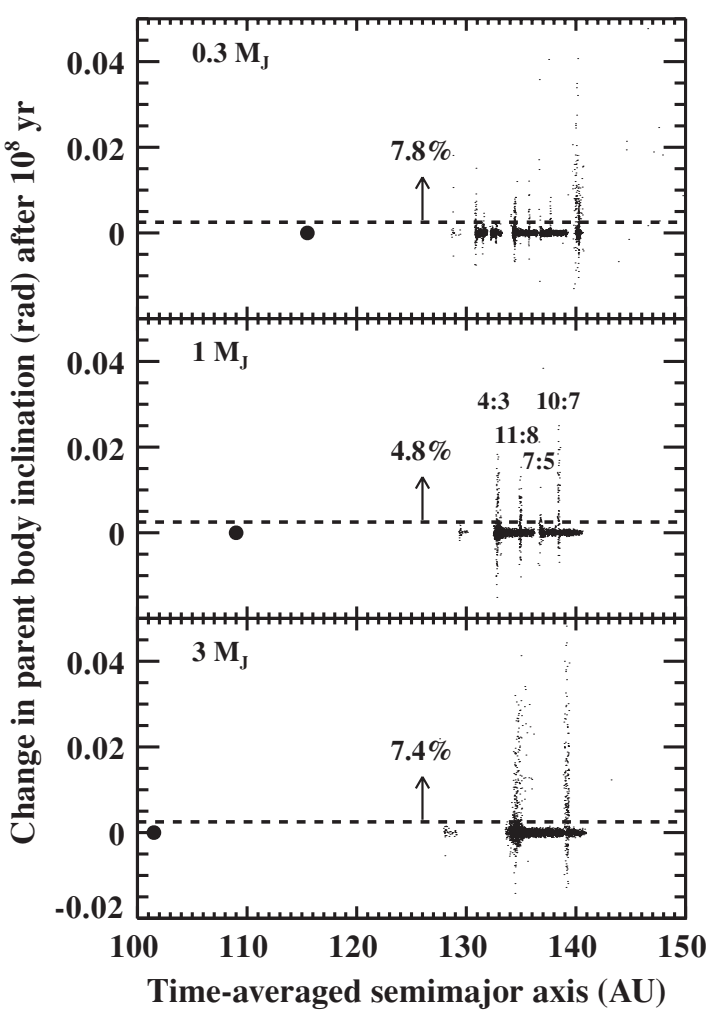

Figure 7. Changes in orbital inclinations of surviving parent bodies, evaluated after $10^{8} \mathrm{yr}$. Solid circles denote the planet. Only small percentages of stable parent bodies have their inclinations increased by the planet by more than $2.5 \times 10^{-3} \mathrm{rad}\left(0.14^{\circ}\right)$; these percentages are indicated in each panel. For comparison, the initial distribution of inclinations extends uniformly from 0 to $2.5 \times 10^{-2} \mathrm{rad}$. Those few objects that have their inclinations amplified are localized to mean-motion resonances, labeled only for the middle panel.

these radiation-induced free eccentricities of dust grains are much larger than the free eccentricities acquired by parent bodies, they do not necessarily lead to increased blurring of the belt inner edge, because the semimajor axes of dust grains shift correspondingly outward by radiation pressure as well (see Equation (5)). We have verified that the various $N_{\beta}$ surface density profiles for $M_{\mathrm{pl}} \leqslant 1 M_{\mathrm{J}}$ have comparably sharp inner edges for all $\beta$, as gauged using our fractional width parameter $\delta$. For further discussion of what influences the sharpness of the inner edge, see Section 3.3.1.

Finally, in Figure 7, we examine how the orbital inclinations of stable parent bodies change after $10^{8} \mathrm{yr}$. L-L predicts that the mutual inclination between the planet and particle is conserved, and indeed most inclinations are little altered from their initial values (which span up to $0.025 \mathrm{rad}$ ). Fewer than $10 \%$ of all surviving parent bodies have final inclinations that exceed initial inclinations by more than $0.0025 \mathrm{rad}$. Excited bodies are localized to mean-motion resonances. Even in the vicinity of a resonance, the fraction of bodies that have their inclinations pumped is small. For example, in our $1 M_{\mathrm{J}}$ model, only $10 \%$ of all stable parent bodies having time-averaged semimajor axes between 132 and $133.1 \mathrm{AU}$-particles right at the inner edge of the belt, in the vicinity of the $4: 3$ resonance-have final inclinations exceeding initial inclinations by more than $0.0025 \mathrm{rad}$. The near constancy of inclination should simplify future modeling efforts: whatever vertical thickness of the belt is desired to match the scattered light images can be input into the dynamical model as an initial condition, in Step 1.

\subsection{Extensions and Refinements}

In Section 3.3.1, we reconcile our results on the sharpness of the inner belt edge with those of Q06. In Section 3.3.2, we examine how robust our optical depth profiles are to uncertainties in $t_{\mathrm{col}}$ and to our simplifying assumption that the profile is adequately simulated by releasing grains from parent bodies at a single time. In Section 3.3.3, we experiment with different test particle initial conditions to see whether they might yield superior fits to the observations.

\subsubsection{Sharpness of Inner Belt Edge}

In Figure 3, we found that the sharpness of the belt's inner edge, as gauged by our measure $\delta$ (see Section 3.2.2), hardly varied with $M_{\mathrm{pl}} \leqslant 1 M_{\mathrm{J}}$. This finding is seemingly at odds with that of Q06, who gauged sharpness using the velocity dispersion of parent bodies at the boundary of the chaotic zone, and who found that it smoothly increases as $\mu^{3 / 7}$. As a consequence of this relation, Q06 concluded that $M_{\mathrm{pl}}$ cannot exceed $\sim 7 \times 10^{-5} M_{*}=0.2 M_{\mathrm{J}}$ and still have the belt edge be as sharp as the observations imply. Indeed, we also found in Figure 6 that the velocity dispersion of parent bodies, as indicated by their spread in free eccentricities, increased smoothly with $M_{\mathrm{pl}}$, in apparent agreement with Q06. Yet the smoothly growing velocity dispersion is not reflected in the relative sharpness of our optical depth profiles across mass models.

How can this be? It might be thought that the discrepancy arises because Q06's calculation of the velocity dispersion pertains to parent bodies, while our calculation of $\tau_{\perp}$ involves dust. While as a point of principle our calculation would be preferred because the observations are of dust and not of parent bodies, this explanation does not get at the heart of the problem, as we find the same invariant sharpness with planet mass characterizing the surface density profiles of our parent bodies.

The answer instead is that the sharpness of the inner edge does not depend only on the velocity dispersion of particles located strictly at the chaotic zone boundary. Particles located at some radial distance from the boundary, further interior to the belt, also contribute to the sharpness. This is because sharpness is a relative quantity, measured relative to the maximum of $\tau_{\perp}$, and this maximum does not occur exactly at the chaotic zone boundary. Sharpness is appropriately measured as a relative quantity, since the observations are limited in a dynamic range: as Figure 3 of K05 indicates, only the maximum in surface brightness and values greater than $\sim 10 \%$ of the maximum are measurable.

To illustrate our point, in Figure 8, we plot the surface densities of stable parent bodies for two mass models, $0.3 M_{\mathrm{J}}$ and $10 M_{\mathrm{J}}$. For the comparison with Q06 to be fair, we must analyze only the parent bodies, since the conclusions of Q06 regarding inner edge sharpness pertain to collisionless, radiation-free particles; in other words, the test particles of Q06 are our parent bodies. From Q06, we would expect that the $10 M_{\mathrm{J}}$ model produces an inner edge that is $(10 / 0.3)^{3 / 7}=4.5 \times$ more diffuse, but the bottom panel of Figure 8 shows that this is not the case; in fact, if anything, the $0.3 M_{\mathrm{J}}$ profile appears more diffuse. In the top panel of Figure 8, we show the same two models except that we include only particles having the smallest stable semimajor axes: $a<132 \mathrm{AU}$ for $M_{\mathrm{pl}}=0.3 M_{\mathrm{J}}$ and $a<136 \mathrm{AU}$ for $M_{\mathrm{pl}}=10 M_{\mathrm{J}}$. These profiles, which more strictly sample the chaotic zone boundary, are more consistent with Q06- the $0.3 M_{\mathrm{J}}$ profile is sharper than the $10 M_{\mathrm{J}}$ profile. We conclude that sharpness cannot be reliably computed without including 


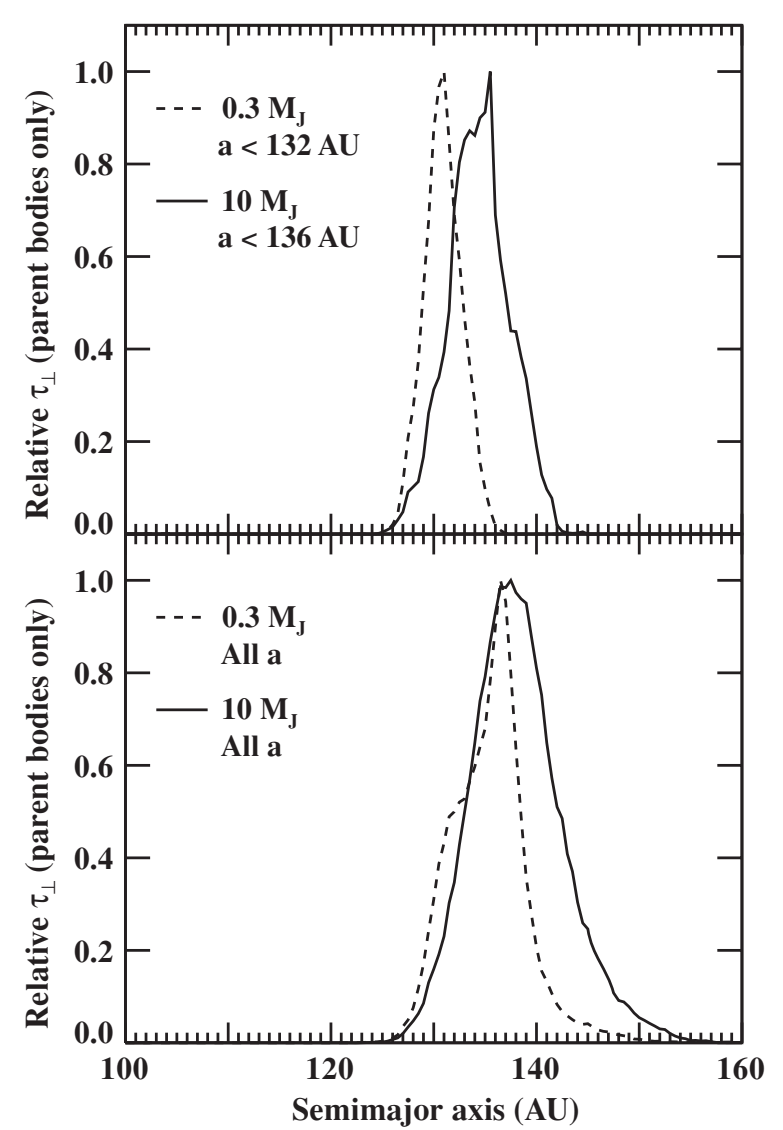

Figure 8. Reconciling our findings on the sharpness of the belt inner edge with those of Q06. Top panel: surface density profiles of stable parent bodies for two mass models, $0.3 M_{\mathrm{J}}$ and $10 M_{\mathrm{J}}$, including only those bodies having the smallest semimajor axes as indicated (see Figure 5). Only parent bodies are considered to compare fairly with Q06, whose conclusions regarding inner edge sharpness pertain to collisionless, radiation-free test particles. In qualitative agreement with $\mathrm{Q} 06$, the $10 M_{\mathrm{J}}$ model yields a more diffuse inner edge, a consequence of that model's larger velocity dispersion at the chaotic zone boundary (see Figure 6). Bottom panel: surface density profiles of the same two models with no restriction on semimajor axes. Now the inner edges are of similar sharpness - in fact, the $10 M_{\mathrm{J}}$ profile appears slightly sharper than the $0.3 M_{\mathrm{J}}$ model —indicating that sharpness is not uniquely related to edge velocity dispersion, in contrast to the implicit assumption of Q06.

contributions from particles that are located some distance from the edge. Sharpness cannot be calculated as if it were a local quantity specific to the minimum stable semimajor axis; the shape of the observed surface brightness profile reflects an amalgam of semimajor axes.

\subsubsection{Variations in $t_{c o l}$ and Gradual Release of Grains}

According to our standard procedure, grain surface densities and optical depths from our $\beta$-simulations are extracted after an integration time of $t_{\mathrm{col}}=10^{5} \mathrm{yr}$, a value inspired from our order-of-magnitude estimate (9) of the collisional lifetime. In the top and middle panels of Figure 9, we demonstrate that our results are not sensitive to uncertainties in $t_{\mathrm{col}}$. We present test results for $\beta=0.2$ and $\beta=0.4$ since those cases contribute most to $\tau_{\perp}$ for our assumed Dohnanyi size distribution. Varying $t_{\text {col }}$ from $3 \times 10^{4} \mathrm{yr}$ to $3 \times 10^{5} \mathrm{yr}$ produces practically identical results for the surface density of grains. Only for the $\beta=0.2$, $t_{\mathrm{col}}=3 \times 10^{5} \mathrm{yr}$ run is there a slight $\sim 1 \mathrm{AU}$ shift of the surface density profile, a consequence of PR drag. Such drag affects $\beta<0.2$ grains even less. The highest $\beta$ grains are also relatively immune from pericenter decay — see the middle panel of Figure 9 for the case $\beta=0.4$-because of the

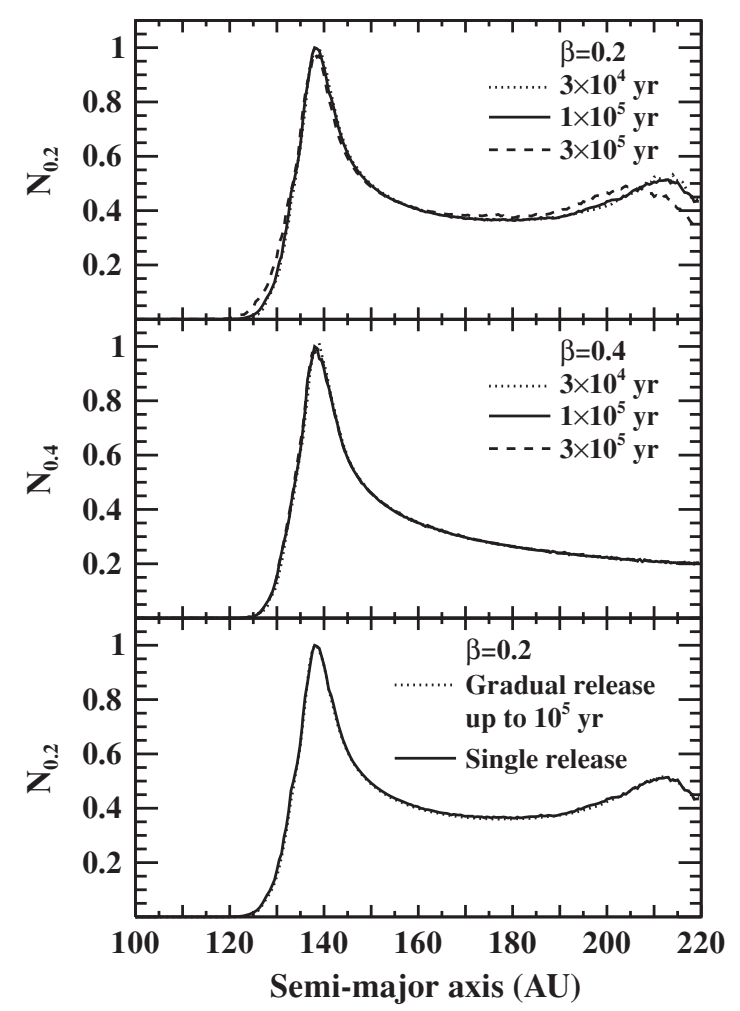

Figure 9. Top panel: surface density profiles for $\beta=0.2$ particles in our $1 M_{\mathrm{J}}$ model, as a function of $t_{\mathrm{col}}$, whose value we only know to order of magnitude. Aside from a small inward shift caused by PR drag for $t_{\text {col }}=3 \times 10^{5} \mathrm{yr}$, the profiles are not sensitive to our uncertainty in $t_{\mathrm{col}}$. Middle panel: same as the top panel, except for $\beta=0.4$. Bottom panel: testing our simplifying assumption of a single release time for dust grains. Allowing grains to be released at 10 uniformly spaced times between $t=0$ and $t=10^{5} \mathrm{yr}$ produces no discernible difference from our standard model, which assumes a single release time of $t=0$.

large eccentricities and semimajor axes induced by radiation pressure upon release (Wyatt \& Whipple 1950, see also our Equation (10)).

We can also check whether our simple procedure of releasing grains at a single time is sound. In reality, the belt at any given moment will contain grains having a variety of ages, ranging from 0 (just released grains) to $t_{\mathrm{col}}$ (grains just about to be shattered to sizes small enough to be blown out by radiation pressure). We better simulate the gradual release of grains by adding together surface density profiles computed from grains released at multiple times. We divide the interval $t_{\mathrm{col}}$ into 10 release times, uniformly spaced by $\Delta t=t_{\mathrm{col}} / 10$, and generate separate integrations for each time. That is, for the $i$ th release time, we integrate the stable parent bodies (generated from Step 1) forward with $\beta=0$ for $i \Delta t$, and then continue integrating the released dust grains with $\beta \neq 0$ for $(10-i) \Delta t$. The resulting superposition of integrations, for $\beta=0.2$ and $t_{\text {col }}=10^{5} \mathrm{yr}$, is shown in the bottom panel of Figure 9; it is nearly indistinguishable from our standard result. We have checked that the ring shape is independent of the grain age $t$ for $1 / \Omega \ll t<t_{\text {col }}$, because $t_{\text {col }}$ is too short a time for grains to evolve away from their birth orbits (say by PR drag), and $1 / \Omega$ is the timescale for grains to phase mix.

The experiments described in all three panels of Figure 9 also show that our standard surface density and optical depth profiles do not reflect dust features that vary with the orbital phase of the planet. In varying $t_{\mathrm{col}}$ and superposing snapshots taken at different times, we extract surface densities corresponding to random orbital phases of the planet. 


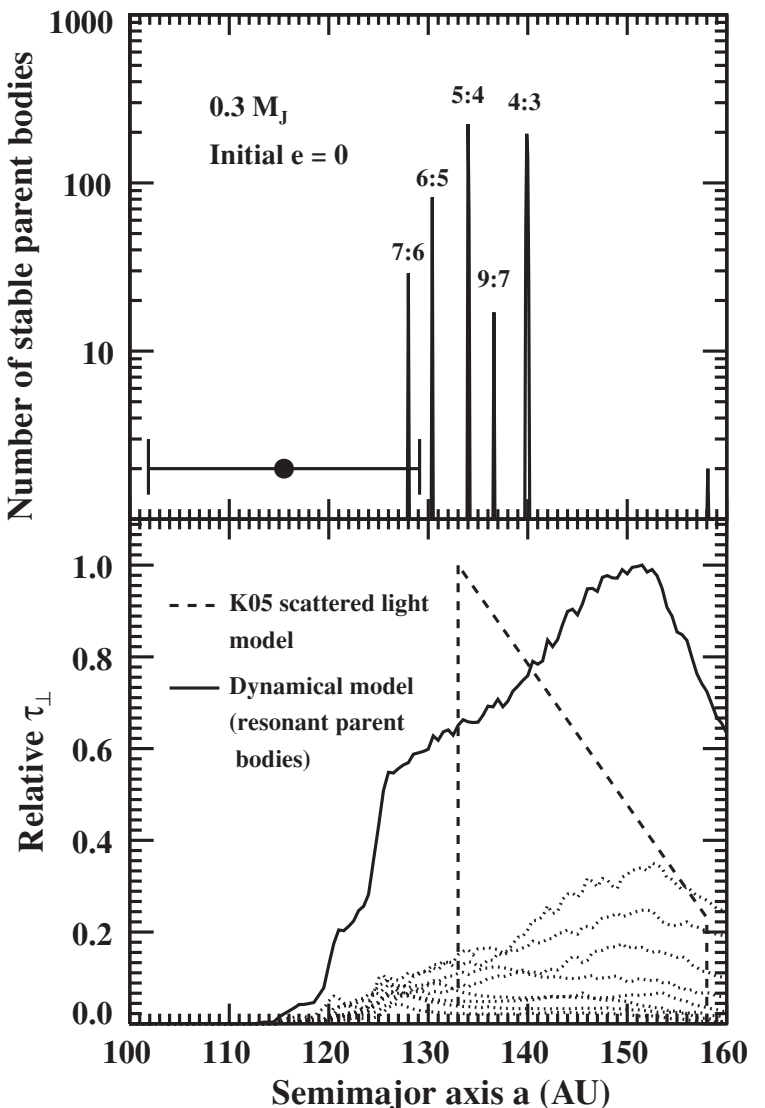

Figure 10. Experimenting with initially zero eccentricities for parent bodies, for the case $M_{\mathrm{pl}}=0.3 M_{\mathrm{J}}$. Top panel: histogram of semimajor axes of parent bodies that survive for $10^{8} \mathrm{yr}$, time averaged over $10^{5} \mathrm{yr}$. In stark contrast to our standard model (Figure 5), survivors only inhabit mean-motion resonances, which afford them protection from the close planetary encounters that would otherwise result from the large eccentricities that develop secularly. Bottom panel: optical depth profile of dust generated by resonant parent bodies. It is far too broad to agree with the K05 model. We conclude that while resonant parent bodies can exist in principle, their population in reality must be small compared with that of parent bodies on nearly purely secularly forced orbits.

\subsubsection{Resonant Particles as Another Class of Stable Parent Body}

All our parent bodies are initialized with purely forced eccentric orbits, as calculated using the L-L secular theory. Their orbits after $10^{8} \mathrm{yr}$ resemble their initial ones, with the addition of a small free component.

Here, we try a different set of initial conditions: osculating $e=0$. According to $\mathrm{L}-\mathrm{L}$, this corresponds to assigning particles free eccentricities equal in magnitude to their forced eccentricities (see, e.g., Murray \& Dermott 2000). The top panel of Figure 10 documents the resultant time-averaged semimajor axes of stable parent bodies (those that survive for $10^{8} \mathrm{yr}$ ), for the case $M_{\mathrm{pl}}=0.3 M_{\mathrm{J}}$. All other initial conditions apart from the test particles' eccentricities are specified in the same way as for our standard $0.3 M_{\mathrm{J}}$ model. Remarkably, comparing the top panels of Figures 10 and 5, we find that the distributions of stable semimajor axes are nearly complementary. Instead of being cleared out of mean-motion resonances, stable parent bodies inhabit them exclusively when initial eccentricities equal zero.

The resonances-which include the $7: 6,6: 5,5: 4,9: 7$, and 4:3, and which are of eccentricity type-protect the particles from close encounters with the planet. Qualitatively, the eccentricities and apsidal angles behave as L-L predicts: while $e$ cycles from

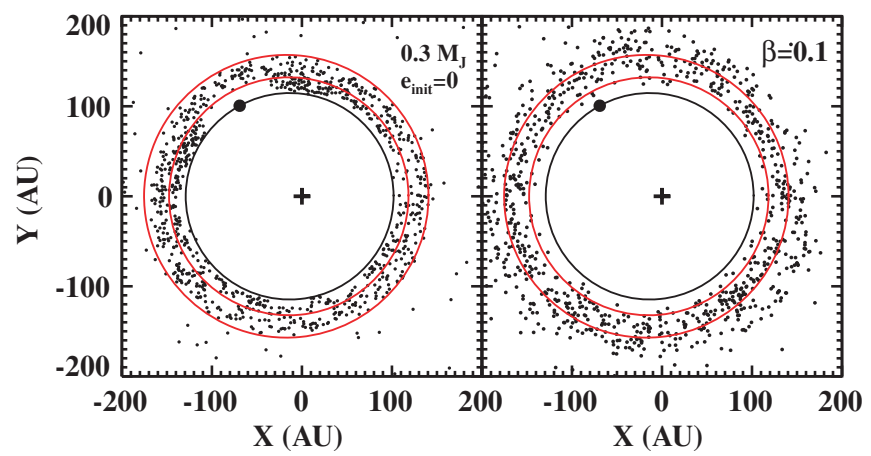

Figure 11. Same as Figure 1, except for resonant parent bodies. The left panel shows how resonant parent bodies avoid close encounters with the planet. The entire pattern corotates with the planet. Dust grains released from these resonant parent bodies have large eccentricities and yield an optical depth profile too broad to match that of K05; see Figure 10.

0 through $\max (e)=2 e_{\text {forced }} \approx 0.22$ back to $0, \tilde{\omega}$ regresses from $\pi / 2$ to $-\pi / 2$ (the evolution of $\widetilde{\omega}$ is discontinuous since $e$ passes through 0 ). The large maximum eccentricities, which result because the free eccentricities are of the same magnitude as the forced eccentricities, put particles in danger of close planetary encounters, especially when $e=\max (e)$ and $\tilde{\omega}=0$. Under these conditions, for a semimajor axis of, say, $a=128 \mathrm{AU}$, the particle's pericenter encroaches within $\sim 2 \mathrm{AU} \approx 0.5 R_{\mathrm{H}}$ of the planet's pericenter.

However, thanks to resonance, conjunctions occur only at special orbital phases and close encounters do not occur. For the circumstances just described, during the phase that the particle attains maximum eccentricity, we have observed in our numerical integrations that conjunctions never occur at periastron. Because of the 7:6 resonance, they occur instead $180^{\circ}$ away, at apoapse, when the bodies are well separated by about 27 AU. Figure 11 provides a snapshot of stable resonant parent bodies, showing how they avoid approaching the planet.

Can such resonant parent bodies be present in Fomalhaut's belt? Not in significant numbers compared with the nonresonant population. The resonant bodies develop more eccentric orbits, and consequently the dust they produce is more spatially extended. From the bottom panel of Figure 10 (see also the right-hand panel of Figure 11), it is clear that the optical depth profile of dust released from resonant parent bodies is far too broad to match that of K05. (Our procedure of calculating optical depths by smoothing particles over their orbits is not correct for resonant objects, since the smoothing ignores their special orbital phase relationships with the planet. However, the error accrued is small, since $\tau_{\perp}$ is dominated by dust particles having $\beta \gtrsim 0.1$. Such dust particles, upon release, have their semimajor axes increased by $\gtrsim 10 \%$ by radiation pressure, and are thus removed from the resonances inhabited by their parents.)

In Section 4.3, we discuss how the parent bodies might have come to occupy nearly purely secularly forced orbits and to avoid the resonant orbits.

\section{SUMMARY AND DISCUSSION}

We review our main results in Section 4.1, sketch the effects of other planets apart from Fom $\mathrm{b}$ in Section 4.2, and discuss possible origins of Fom $b$ and the belt in Section 4.3.

\subsection{Summary}

Fomalhaut $\mathrm{b}$ is the first extrasolar planet candidate to be directly imaged at visible wavelengths and to have its orbital 
motion around its host star measured. Surprises have been immediate: Fom b has an unusually large orbital radius of more than 110 AU (see Lafrenière et al. 2008) and a visual (0.45$0.7 \mu \mathrm{m}$ ) luminosity that is not only $1-2$ orders of magnitude greater than atmospheric models anticipated, but also time variable. Nevertheless, a chain of arguments based on comparing the observed photometry with model exoplanet atmospheres leads K08 to infer that the mass of Fom b must be less than about $3 M_{\mathrm{J}}$.

The Fomalhaut system is all the more remarkable for offering a means independent of model spectra to get at Fom b's mass: the star is encircled by a belt of dust whose geometry is, in principle, sensitive to the mass and orbit of Fom b. At a system age of $\sim 200 \mathrm{Myr}$, detritus from the formation of the Fomalhaut planetary system still remains. If Fom b is the sole sculptor of this debris - but see Sections 1 and 4.2 for reasons that it might not be-then the observed intrinsic ellipticity of the ring would owe its origin to secular forcing by Fom b, which itself would reside on an apsidally aligned and similarly eccentric orbit to the belt's (Wyatt et al. 1999; Quillen 2006). Under the single planet assumption, another feature of the belt influenced by Fom $b$ would be its inner edge. The observed sharpness with which the belt truncates would reflect the sharp divide between stability and chaos at the boundary of the planet's chaotic zone, inside of which first-order mean-motion resonances overlap and particle orbits are short-lived (Wisdom 1980; Quillen 2006). Particles inside the zone quickly evolve onto planet-crossing orbits and thereafter are perturbed onto escape trajectories. ${ }^{10}$ Identifying the belt inner edge with the chaotic zone boundary relates the distance between the planet and the belt edge to the planet-star mass ratio.

Based on these and other theoretical ideas, we have built a realistic dynamical model of the Fomalhaut planet-belt system, under the single planet assumption. Our goal is to calculate the spatial distribution of dust generated from the collisional comminution of larger parent bodies, and to compare our dust maps with the HST scattered light observations. The model begins by using numerical integrations to establish an annulus of parent bodies - aka the "birth ring" (Strubbe \& Chiang 2006) that is stable for $100 \mathrm{Myr}$ against perturbations by Fom b. Dust particles are released from these dynamically stable parents, and their trajectories followed, taking additional account of stellar radiation forces, for a dust collisional lifetime of $t_{\mathrm{col}}=0.1 \mathrm{Myr}$. The orbits of dust particles at the end of a $t_{\mathrm{col}}$-long integration are assumed to well represent those of actual dust particles, which in reality have a range of ages extending up to $\sim t_{\mathrm{col}}$. We have subjected this assumption to a few tests and found it to hold. Final dust particle orbits, computed for a range of radiation $\beta$ 's (force ratio of stellar radiation pressure to stellar gravity), are converted into orbit-averaged maps of relative surface density (number of grains per unit face-on area of the belt). A Dohnanyi (1969) grain size distribution, appropriate for a quasi-steady collisional cascade, is assumed to hold in the birth ring, where the dust surface density is highest and the collision rate is greatest. This assumption determines how we weight and add the surface density maps to produce a map of (relative) vertical optical depth. This optical depth map-or rather its azimuthally averaged version, the variation of optical depth with the semimajor axis-is compared with the optical depth profile of the Kalas et al. (2005) scattered light model,

\footnotetext{
10 We estimate that planet crossing takes $\sim 10^{5}\left(10^{-3} / \mu\right)^{4 / 7} \mathrm{yr}$ and ejection takes $\sim 10^{7}\left(10^{-3} / \mu\right)^{2} \mathrm{yr}$, for particles halfway between the planet and the edge of the chaotic zone.
}

which itself represents an idealized and approximate fit to the HST images.

A conservative result of our dynamical model is that $M_{\mathrm{pl}}<$ $3 M_{\mathrm{J}}$. This conclusion, that Fom $\mathrm{b}$ must be of planetary mass, is entirely independent of Fom b's photometry and its uncertain interpretation. Our result stems from two simple and robust trends, neither of which involve inner edge sharpness, in contrast to Q06. First, as $M_{\mathrm{pl}}$ increases, dust particles are increasingly perturbed by the planet onto more eccentric orbits, rendering the dynamical profiles too broad compared with the scattered light profile. A mass of $10 M_{\mathrm{J}}$ yields a belt that is about twice as bright between 150 and $160 \mathrm{AU}$ as the observations allow, while lower masses $M_{\mathrm{pl}}<3 M_{\mathrm{J}}$ give optical depth profiles that we feel agree adequately well with the Kalas et al. (2005) scattered light model. Second, given the observed location of the inner edge of the belt, larger mass planets have necessarily smaller orbits located farther interior to the belt, and smaller orbits may be incompatible with the observed stellocentric distance of Fom b. A mass of $10 M_{\mathrm{J}}$ requires an orbit whose apocentric distance is $107 \mathrm{AU}$, falling well short of our estimated deprojected distance for Fom b of $119 \mathrm{AU}$. By contrast, for $M_{\mathrm{pl}} \leqslant 1 M_{\mathrm{J}}$, the model apocentric distances are $\geqslant 122 \mathrm{AU}$. While $3 M_{\mathrm{J}}$ yields an orbit whose apocenter lies at $115 \mathrm{AU}$ and is nominally incompatible, uncertainties in the observed deprojected distance, probably amounting to a few AU, preclude us from ruling out this mass. Thus, erring on the safe side, we conclude that $M_{\mathrm{pl}}<3 M_{\mathrm{J}}$.

Our findings agree in broad outline with those of Quillen (2006), insofar as we also identify the inner belt edge with the boundary of the planet's chaotic zone. We diverge from Quillen (2006) in how we determine Fom b's mass. Some of the improvements that we make are as follows: (1) we draw a clear distinction between unobservable parent bodies and observable dust grains, and rely on the latter when comparing with the HST scattered light observations; (2) we include the effect of stellar radiation pressure, significant for dust grains; (3) parent bodies are screened for dynamical stability over the age of the system; and (4) grain-grain collisions are recognized as destructive and, therefore, the duration of each of our dust particle integrations is necessarily limited by the collision time. Our upper mass limit of $3 M_{\mathrm{J}}$ follows, in part, from comparing theoretical and observed optical depth profiles of dust, computed globally over all space, and from noting how steep those profiles are outside of the location of peak optical depth. By contrast, the upper mass limit derived by Quillen $(2006), \sim 0.2 M_{\mathrm{J}}$, follows from an analysis of the radiation-free dynamics of collisionless particles - essentially, the dynamics of parent bodies-local to the chaotic zone boundary, and from the assumption that the local velocity dispersion of such bodies determines the sharpness of the inner belt edge. Our calculation of the upper mass limit is preferred because the HST observations are of dust, and not of parent bodies, and also because we have shown that edge sharpness is better computed using a global model such as ours.

Three Jupiter masses for the mass of Fom $b$ is an upper limit in still another sense: our results are based on the assumption that Fom $b$ alone sculpts the belt. Roughly speaking, the more planets that are present, the more the orbits are chaotic, and the more effectively small bodies and dust are gravitationally scoured. Thus, the observed inner edge of Fomalhaut's belt is compatible with a mass for Fom $b$ that is lower than that computed under the single planet assumption. It is heartening to see that our preferred mass range of $M_{\mathrm{pl}}<3 M_{\mathrm{J}}$ supports that inferred from the spectral models. 
Perhaps the biggest deficiency of our model lies in our crude treatment of grain collisions. In setting a dust particle's initial position and velocity equal to that of its parent body, we ignore collisional dissipation and redirection of orbital kinetic energy. We also neglect the fact that grinding the largest parent bodies down to dust requires multiple collisions, and that radiation effects can start manifesting in the middle of the collisional cascade. For example, a particle for which radiation effects are significant, say which has $\beta=0.4$, can be born from a parent body for which radiation effects were also significant, say which had $\beta=0.2$. Still, our simple prescription of releasing dust grains having $\beta>0$ from bodies having $\beta=0$ is not without justification. Collisional energy dissipation should, on average, dampen free eccentricities but not forced eccentricities (see Section 4.3); thus, the mean elliptical shape of the belt is expected to be preserved. Grain ejection velocities relative to the parent are distributed isotropically and should therefore not bias our results, though they will produce larger free eccentricities than our model predicts. Larger free eccentricities will result in smoother optical depth profiles: a blurring of the belt (see Section 4.3 for further discussion of free eccentricities). Finally, radiation effects are predominantly felt over only the last decade in grain size above the blow-out size, that is, only after the penultimate collision just prior to the final collision resulting in blow-out, for collisions that are strongly disruptive. The next generation of models should test these assertions, in addition to considering qualitatively different physics (e.g., Yarkovsky drag and gas-particle interactions ${ }^{11}$ ).

\subsection{Other Planets in Addition to Fom $b$}

As discussed in Section 1, the observed space velocity of Fom $\mathrm{b}$ is nominally inconsistent with its orbit being apsidally aligned with that of the belt, contradicting a basic prediction of the hypothesis that Fom b is solely responsible for the morphology of Fomalhaut's debris disk. The final word on orbits must await more epochs of astrometry and more realistic assessments of systematic uncertainties (in, e.g., frame registration between epochs and stellar mass). Even if the apsidal misalignment proves real, and even if follow-up observations constrain Fom b's mass to be less than that required to make a significant contribution to shaping the belt, ideas of chaotic zone clearing and secular forcing of eccentricity may still be relevant for the Fomalhaut system, provided there are perturbers in addition to Fom $b$.

We have already argued that multiple planets are compatible with the inner edge to Fomalhaut's debris disk; more planets help eject more material from inside the hole. Multiple planets are also compatible with the observed belt eccentricity. In L-L theory, the observed forced eccentricity vector of a belt particle equals the vector sum of $n$ eccentricity vectors forced by $n$ planets. Because the individual eccentricity vectors precess at frequencies that depend only on the fixed masses and semimajor axes of the planets (these are the fixed eigenfrequencies of the linear theory), and because belt particles having similar semimajor axes have similar vector decompositions, we expect the forced eccentricity vectors of belt members to remain similar to one another over time. Thus, a narrow belt can maintain a global mean eccentricity in the presence of multiple planets, though that mean eccentricity will oscillate with time. All these

\footnotetext{
11 Fomalhaut's closest analog may be AU Mic, insofar as both have birth ring morphologies and similar optical depths (Strubbe \& Chiang 2006). Gas has not been detected in AU Mic (Brandeker et al. 2008, Spitzer Science Conference Poster \#81).
}

considerations can be accommodated as necessary into our modeling procedure.

If future astrometry confirms a significant apsidal misalignment between the planet and belt, then at least one other, as yet unseen, planet would be implicated. In that case, because the belt's forced eccentricity is a vector sum, Fom b's eccentricity could either be lower or higher than the eccentricity we have calculated, depending on the apsidal orientation(s) of the other planetary orbit(s).

Intriguingly, over its three year mission, the Hipparcos satellite observed Fomalhaut to have an "anomalous" proper acceleration of 6.6 mas yr $^{-2}$, of marginal (about $2 \sigma$ ) significance. Such a quasi-steady acceleration might be caused by a companion whose orbital period is longer than $\sim 3$ years, or equivalently whose stellocentric distance $r \gtrsim 3$ AU. Equating the measured acceleration with $\left(G M / r^{2}\right) / d$, where $M$ is the perturbing mass and $d=7.7 \mathrm{pc}$ is the distance to Fomalhaut, we see that Fomalhaut might also be harboring a $\sim 30 M_{\mathrm{J}}$ brown dwarf at a distance $r \sim 5 \mathrm{AU}$. (Larger $r$ implies larger $M$ and such solutions are probably ruled out by observation. Clearly, Fom $b$ cannot be responsible for the Hipparcos acceleration.) Compared against the influence of Fom $b$ as we have computed it in this paper, such a brown dwarf would contribute more than $10 \%$ to the forced eccentricity of a belt particle, if the brown dwarf's eccentricity $\gtrsim 0.2$.

The above lines of evidence for additional planets perturbing the belt are tenuous. Given the observed proximity of Fom b to the belt, the opposing case can be made that Fom b dominates the forced eccentricities of belt particles. A possible analogy would be Neptune and the Kuiper belt. Despite the existence of as many as four giant planets in our solar system, the forced inclination and eccentricity vectors of Kuiper belt objects are largely determined by the nearest planet, Neptune (see, e.g., Chiang \& Choi 2008). If Fom b's orbit is apsidally aligned with the belt, then Fom b's observed deprojected stellocentric distance of 119 AU (Section 3.2.3) and current true anomaly of about 109 degrees would imply a semimajor axis of 114115 AU. Given such a semimajor axis, our model would predict $M_{\mathrm{pl}}=0.5 M_{\mathrm{J}}($ Table 1$)$.

\subsection{Parent Body and Planet Origins}

Though the direct detection of parent bodies is beyond the reach of current observations, our study has provided some evidence that they reside mostly on nearly purely secularly forced orbits with small free eccentricities. In principle, they do not have to; we found by experimentation in Section 3.3.3 that large free eccentricities are also compatible with longterm stability if the particles are protected by mean-motion resonances.

How did the parent bodies choose one class of stable orbit over the other? The answer, as also suggested by Quillen \& Faber (2006), likely involves collisional dissipation. Collisions dissipate random orbital motions and compel planetesimals to conform toward closed, nonintersecting orbits viewed in the frame rotating with the perturbation potential (e.g., Paczynski 1977; Goldreich \& Tremaine 1982, Section 5.4). If that perturbation potential arises from Fom b, the special closed orbits include the secularly forced orbits that we have been highlighting throughout our study, but they do not include the resonant orbits that emerged from our experiment. Collisional dissipation and relaxation onto closed orbits occur across the entire collisional cascade, up to the largest parent bodies (which by definition collide once over the system age). 
We do not expect the destructive nature of collisions to qualitatively alter this picture, since what is important here is the dissipation of random kinetic energy, and that occurs whether or not collisions are destructive. Postcollision fragments will have free eccentricities that are small compared with forced eccentricities, insofar as postcollision fragment velocities (measured relative to the center of mass) are small compared to $e_{\text {forced }} \Omega_{R} R \sim$ $400 \mathrm{~m} \mathrm{~s}^{-1}$. At least in the collisional genesis of the Eunomia and Koronis asteroid families in our solar system's main belt, ejection velocities of the largest postcollision remnants range from 4 to $90 \mathrm{~m} \mathrm{~s}^{-1}$ (Michel et al. 2001).

It is also possible that relaxation occurred while the parent bodies were forming, when collisions were gentler and agglomerative. The process of relaxation onto forced orbits can be explored using fast numerical simulation techniques for inelastically colliding, indestructible particles (Lithwick \& Chiang 2007); in fact, we have started to run such simulations and clearly observe relaxation.

What are the origins of Fom $b$ and the belt? It seems likely that the belt is what remains of the original disk material that went into building Fom b. If we take the minimum parent body mass of $3 M_{\oplus}$ (estimated in Section 2.6) and augment it by a factor of $10^{2}$ to bring it to cosmic composition, then the minimum primordial mass for the belt is $\sim 1 M_{\mathrm{J}}$. This is comparable to our upper mass limit for Fom b. A working hypothesis is that Fom $b$ accreted in situ from a primordial disk of gas and dust, that the hydrogen gas of the original disk has either accreted into planets or photoevaporated, and that today the remaining solids in the belt are grinding down to dust, the in-plane velocity dispersion of parent bodies excited so strongly by Fom b, and possibly other perturbers, that collisions are destructive rather than agglomerative. The last tenet is supported by our Figure 6, which shows free eccentricity dispersions that imply relative parent body velocities upward of $\sim 100 \mathrm{~m} \mathrm{~s}^{-1}$ (see also our Section 2.3).

This work was supported by NSF grant AST-0507805. E.K. acknowledges support from a Berkeley Fellowship. We thank M. Fitzgerald, Y. Lithwick, E. Mamajek, C. Marois, N. Murray, K. Stapelfeldt, and M. Wyatt for discussions. An anonymous referee rapidly provided a thoughtful report, at the behest of ApJ Scientific Editor F. Rasio, for which we are grateful.

\section{REFERENCES}

Backman, D. E., \& Paresce, F. 1993, in Protostars and Planets III, ed. E. H. Levy \& J. I. Lunine (Tuscon, AZ: Univ. Arizona Press), 1253

Barrado y Navascues, D. 1998, A\&A, 339, 831

Barrado y Navascues, D., Stauffer, J. R., Hartmann, L., \& Balachandran, S. C. 1997, ApJ, 475, 313

Burns, J. A., Lamy, P. L., \& Soter, S. 1979, Icarus, 40, 1

Burrows, A., Sudarsky, D., \& Lunine, J. I. 2003, ApJ, 596, 587

Canup, R. M., \& Ward, W. R. 2002, AJ, 124, 3404

Chiang, E., \& Choi, H. 2008, AJ, 136, 350

Dohnanyi, J. W. 1969, J. Geophys. Res., 74, 2531

Duncan, M., Quinn, T., \& Tremaine, S. 1989, Icarus, 82, 402

Fortney, J. J., Marley, M. S., Saumon, D., \& Lodders, K. 2008, ApJ, 683, 1104

Goldreich, P., \& Tremaine, S. 1982, ARA\&A, 20, 249

Holland, W. S., et al. 1998, Nature, 392, 788

Hubickyj, O., Bodenheimer, P., \& Lissauer, J. J. 2005, Icarus, 179, 415

Kalas, P., Graham, J. R., \& Clampin, M. 2005, Nature, 435, 1067

Kalas, P., et al. 2008, Science, 302, 1345, (K08)

Lafrenière, D., Jayawardhana, R., \& van Kerkwijk, M. H. 2008, ApJ, 689, L153

Lithwick, Y., \& Chiang, E. 2007, ApJ, 656, 524

Marois, C., Macintosh, B., \& Barman, T. 2007, ApJ, 654, L151

Michel, P., Benz, W., Tanga, P., \& Richardson, D. C. 2001, Science, 294, 1696

Mosqueira, I., \& Estrada, P. R. 2003, Icarus, 163, 198

Murray, C. D., \& Dermott, S. F. 2000, Solar System Dynamics (Cambridge: Cambridge Univ. Press)

Paczynski, B. 1977, ApJ, 216, 822

Quillen, A. C. 2006, MNRAS, 372, L14

Quillen, A. C. 2007, MNRAS, 377, 1287

Quillen, A. C., \& Faber, P. 2006, MNRAS, 373, 1245

Song, I., Caillault, J.-P., Barrado y Navascués, D., \& Stauffer, J. R. 2001, ApJ, 546,352

Strubbe, L. E., \& Chiang, E. I. 2006, ApJ, 648, 652

Thébault, P., \& Wu, Y. 2008, A\&A, 481, 713

Wisdom, J. 1980, AJ, 85, 1122

Wisdom, J. 1982, AJ, 87, 577

Wisdom, J. 1983, Icarus, 56, 51

Wisdom, J. 1985, Icarus, 63, 272

Wisdom, J., \& Holman, M. 1991, AJ, 102, 1528

Wyatt, M. C. 2005, A\&A, 433, 1007

Wyatt, M. C., Dermott, S. F., Telesco, C. M., Fisher, R. S., Grogan, K., Holmes, E. K., \& Piña, R. K. 1999, ApJ, 527, 918

Wyatt, S. P., \& Whipple, F. L. 1950, ApJ, 111, 134 